\title{
Tecnologie avanzate per la rappresentazione dell'apparecchiatura costruttiva storica: HBIM e il rinnovarsi di un'istanza
}

\author{
Stefano Brusaporci \\ Alessandra Tata \\ Mario Centofanti
}

\section{Abstract}

II tema del rilievo e rappresentazione dell'apparecchiatura costruttiva storica è essenziale nel processo di conoscenza storico critica dei manufatti architettonici. Si tratta di una conoscenza solo in alcuni casi diretta, spesso esito di indagini o informazioni indirette. L'alto livello di iconicità delle restituzioni coniuga conoscenze puntuali con elementi desunti da simili casi di studio. Le procedure HBIM, ontologicamente [I] sviluppate per una gestione informativa dedicata anche ai sistemi costruttivi, rinnovano le istanze di modellazione dell'apparecchiatura costruttiva, coniugando nel processo di gestione delle informazioni tanto oggetti 3D che tradizionali disegni bidimensionali, secondo un processo dove le istanze di 'trasparenza' delle fonti e 'affidabilità' delle informazioni giocano un ruolo di primo piano. II contributo, dopo aver inquadrato il tema di studio, analizza le possibilità offerte dall'HBIM, con particolare riferimento alle opportunità offerte dall'impiego di oggetti parametrici per la modellazione di elementi costruttivi storici.

\section{Parole chiave}

rilievo architettonico, apparecchiatura costruttiva, HBIM, modellazione 3D, vpl.

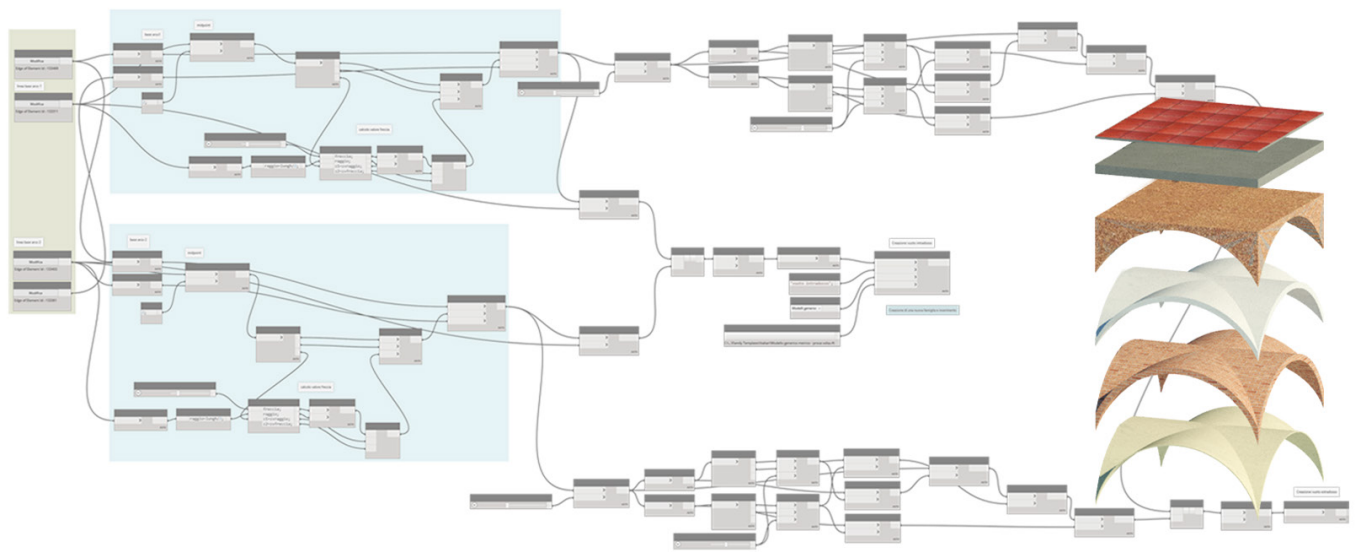




\section{Introduzione}

Nella tradizione delle esperienze italiane, in particolare dalla fine degli anni Ottanta, il tema del rilievo dell'apparecchiatura costruttiva è stato oggetto di specifici approfondimenti da parte delle discipline rivolte al cosiddetto 'built heritage'. In particolare le esperienze dei 'Manuali', dei 'Codici di Pratica', e degli 'Atlanti' [2] riferiscono ad una linea di studio dedicata ai centri storici [3], svolta con una specifica attenzione ai centri minori e all'architettura tradizionale [4]. Una attenzione che ha spostato il fuoco dal monumentale - seppur correlato al suo contesto - ad un approccio inclusivo, a correlare le dimensioni del naturale e dell'antropico, a diverse scale di approfondimento. In questo ambito rientrano le espressioni della cultura costruttiva, secondo una sfera dove il manufatto, inteso come testimoniale del fare umano, assurge a documento principe, spesso anche nell'assenza di significativi riferimenti documentali.

Lo studio, il rilievo e la rappresentazione dell'apparecchiatura costruttiva storica trovano specifici approfondimenti soprattutto nell'ambito delle discipline del Restauro Architettonico [5], del Disegno [6] e dell'Archeologia, quest'ultima con particolare riferimento alle tecniche murarie, tanto antiche che medievali [7]. Se nell'ultimo decennio questo tema sembra piuttosto relegato a specifici contributi locali, d'altro canto si ritiene che le procedure HBIM reclamino una necessaria nuova riflessione, in quanto per prime, nell'ambito delle tecnologie digitali dell'architettura, semanticamente fondate sulla gestione informativa e modellazione digitale dell'apparecchiatura costruttiva.

\section{Rappresentare l'apparecchiatura costruttiva storica}

II tema del rilievo dell'apparecchiatura costruttiva storica è opportunamente affrontato nell'ambito dei principali manuali di rilevamento architettonico [Docci, Maestri 2009; Bertocci, Bini 2012]. La rappresentazione è tradizionalmente condotta con piante e sezioni alle
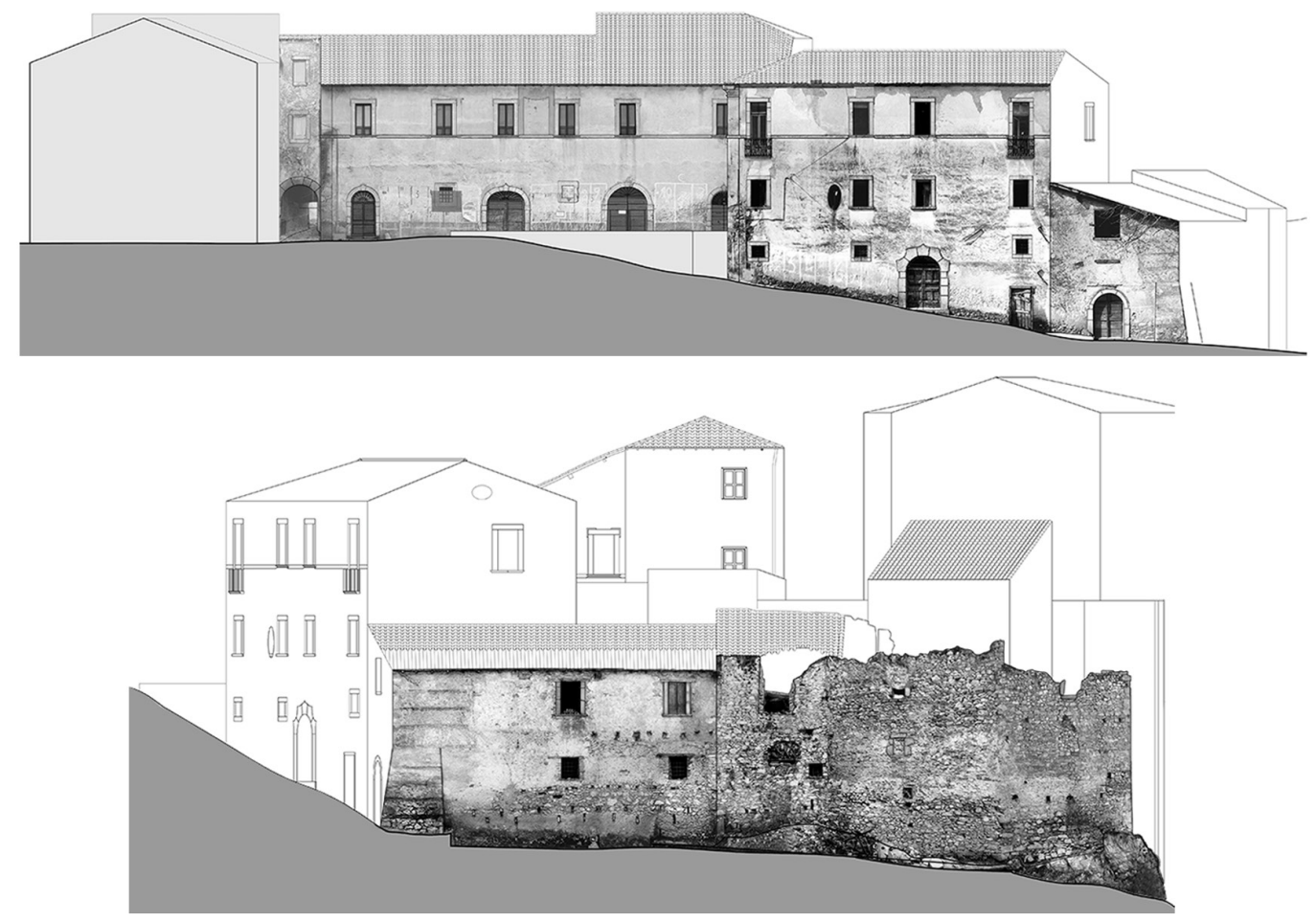
varie scale, ma anche con spaccati ed esplosi assonometrici, utili ad illustrare l'articolazione dei differenti componenti e le loro relazioni [8].

Scrive Carbonara nel numero fondativo della rivista Disegnare. Idee Immagini: "Si tratta quindi di un intendere storicamente e di un disegnare e rilevare criticamente tanto in grande quanto in dettaglio, di un vedere e d'un rappresentare in profondità [...] condotti in primo luogo con l'ausilio di un'attenta ispezione di una frequentazione assidua e personale dell'opera. D'una anticipazione di analisi storico-critica diagnostica attuata vista e 'al tatto', con mezzi relativamente semplice ma con sensibilità ed esperienza la quale ricorda, chiamando in causa il consueto confronto della medicina con restauro, la buona pratica del dottore di famiglia del vecchio medico condotto" [Carbonara 1989, p. 85]. È il "rilievo anatomico" di cui parla Centofanti [Centofanti 20 I0, pp. 43-54], che sviluppa il tema del modello interpretativo, con specifico riferimento alla questione dello studio dell'apparecchiatura costruttiva, anche nelle dimensioni del tridimensionale e del digitale.

La conoscenza dell'apparecchiatura costruttiva è nella maggior parte dei casi una esperienza indiretta, di un sistema complesso che si cela dietro la superficie. Può talvolta essere diretta e completa (almeno per alcune parti), altrove discreta per saggi ed introspezioni, oppure derivare da notizie di archivio o di progetto, infine trattarsi di una ipotesi ricostruttiva, condotta sulla base dell'esperienza e cultura del rilevatore, oppure suggerita da studi o manualistica. Solo nella fase di cantiere, può trovare una epifania. Pertanto quando ci si rapporta con la rappresentazione dell'apparecchiatura costruttiva del costruito, la conoscenza e la rappresentazione è giocoforza in larga parte ricondotta all'utilizzo di simbologie ovvero spesso alla rappresentazione - ed adattamento - di apparecchiature tipologicamente simili, rilevate altrove. Ad esempio nel disegno in sezione di una muratura, questa può essere disegnata con riferimento ad un 'sistema' atto ad evidenziarne le peculiarità (tipologia degli elementi, dimensioni massime, medie e minime, modalità di posa in opera). É un gioco di rimandi tra un disegno di verosimiglianza nella rappresentazione dei componenti, ed interpretazione del sistema costruttivo. Pertanto la 'frammentarietà' della conoscenza diretta riconduce a modelli grafici fortemente delineati nei particolari, ma che solo in parte derivano da misura diretta, e che vogliono descrivere i caratteri significativi di quanto rappresentato, in un gioco tra specificità del manufatto e tipologia costruttiva.

Nei fatti, questo aspetto potrebbe interfacciarsi utilmente con l'approccio per oggetti parametrizzati del BIM. In ogni caso, appaiono essenziali le questioni relative alla 'Trasparenza' (cioè la dichiarazione delle fonti e dei processi di conoscenza) e alla 'Affidabilità' (cioè dei limiti interpretativi geometrici ed informativi) dei componenti costruttivi digitali [Brusaporci 2017; Bianchini, Nicastro 20 I8; Maiezza 2019].

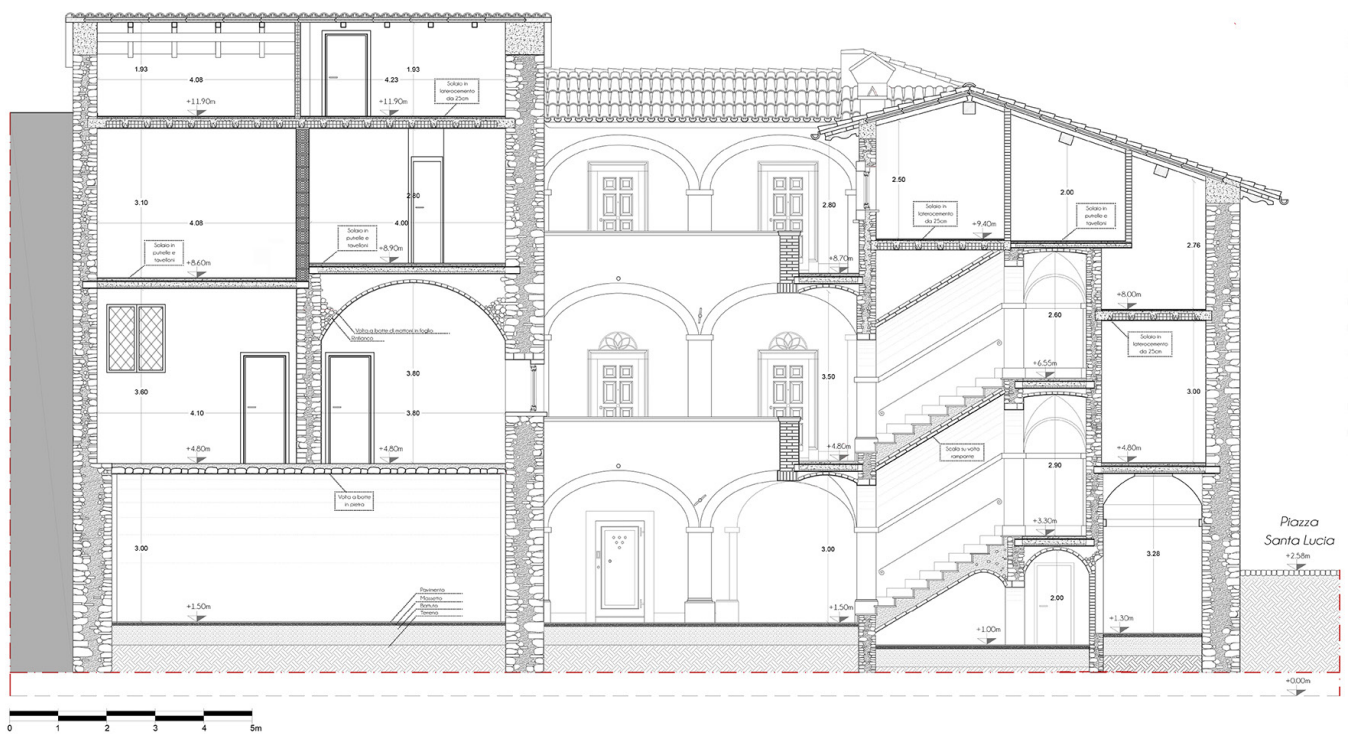


Fig. 3. Rilievo della carpenteria delle coperture di un edificio a Castel del Monte (elaborazione grafica V. Cetra).

Fig. 4. Rilievo dell'apparecchiatura costruttiva di Palazzo Falconi a Torre di Taglio (elaborazione grafica M. Curti).
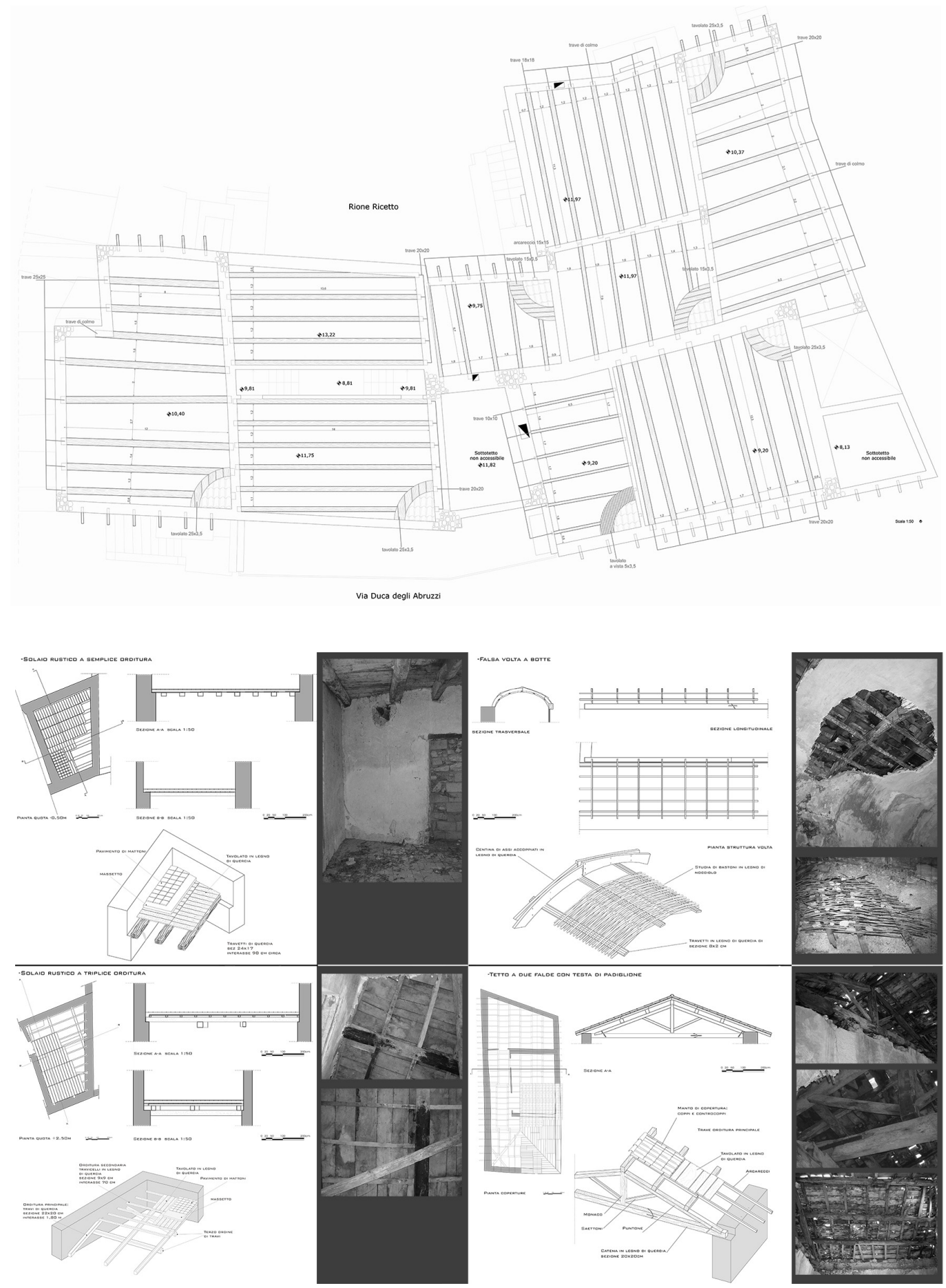


\section{L’apparecchiatura costruttiva storica nei processi HBIM}

II BIM è un processo di gestione di informazioni relative all'edificato. II database è riferito ad un modello 3D, semanticamente definito in relazione all'apparecchiatura costruttiva. Con specifico riferimento alla più recente linea che pone al centro del processo i cosiddetti LOIN (Level of Information Need cioè i livelli informativi richiesti), appare evidente come il modello non venga a costituirsi quale clone sintetico della realtà (tanto nel progetto quanto nel rilievo), ma si pone come modello interpretativo, dove i tradizionali LoD (Livelli di sviluppo) possono, a seconda delle circostanze e finalità, presentare diversa definizione, articolazione e caratterizzazione. Se del caso, infatti il modello può essere arricchito da tradizionali disegni bidimensionali o dati alfanumerici.

Nonostante la stretta relazione tra l'aspetto geometrico e quello informativo all'interno della procedura BIM, ad oggi la maggior parte delle linee di ricerca relative all'utilizzo dell'HBIM è volta o alla rappresentazione geometrico-dimensionale dei componenti edilizi storici o alla documentazione del patrimonio costruito [9]. All'interno di tali sperimentazioni sono però in numero ridotto gli studi che si occupano del tema della rappresentazione dell'apparecchiatura costruttiva degli edifici storici [10].

Fig. 5. Analisi delle apparecchiature murarie di Palazzo Falconi a Torre di Taglio (elaborazione grafica M. Curti).
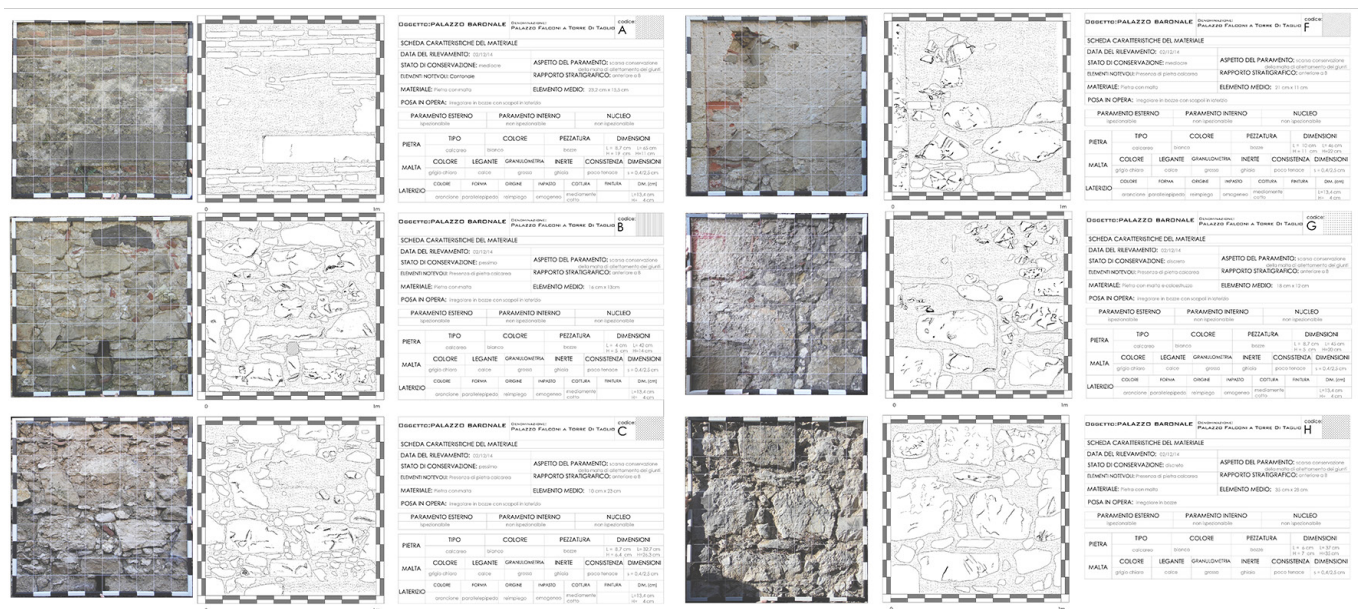

L'importanza dell'aspetto informativo all'interno della logica BIM è sottolineata anche dalle normative vigenti sia italiane che estere. La UNI I I 337:20 I 7 infatti, in accordo con il Sistema britannico PAS I | 92-2 del 20 I3, scinde il livello di sviluppo degli oggetti LoD in: LoG, livello di sviluppo degli oggetti-attributi geometrici, e Lol, livello di sviluppo degli oggetti-attributi informativi.

La conoscenza dell'apparecchiatura costruttiva è perlopiù frammentaria, in genere non esaustiva, derivante da esperienze indirette o dirette. II modello HBIM quindi è costituito da elementi con LoD non uniformi in cui la modellazione dei componenti dipenderà dalle informazioni disponibili e sarà frutto di un processo critico in cui il modellatore, in caso di mancanza di informazioni certe, metterà a disposizione anche la sua esperienza per avanzare ipotesi interpretative. Pertanto le scelte operative devono essere descritte in relazione alla documentazione conoscitiva di base, secondo i noti principi di 'Trasparenza' e 'Affidabilità' degli oggetti del modello [Bianchini, Nicastro 20 I8; Brusaporci et al. 20 I 8]. L'importanza assunta da questi concetti è confermata dalla UNI che definisce il LoD come misurato dalla "natura, quantità, qualità e stabilità dei dati e delle informazioni" associate ad ogni oggetto digitale; al progredire da un LoD a quello successivo quindi è associato un incremento sia della quantità delle informazioni che della loro qualità intesa nel senso di reliability e consolidamento del dato. 
Fig. 6. Modellazione HBIM di un solaio di Palazzo Falconi a Torre di Taglio (elaborazione grafica A. Tata).
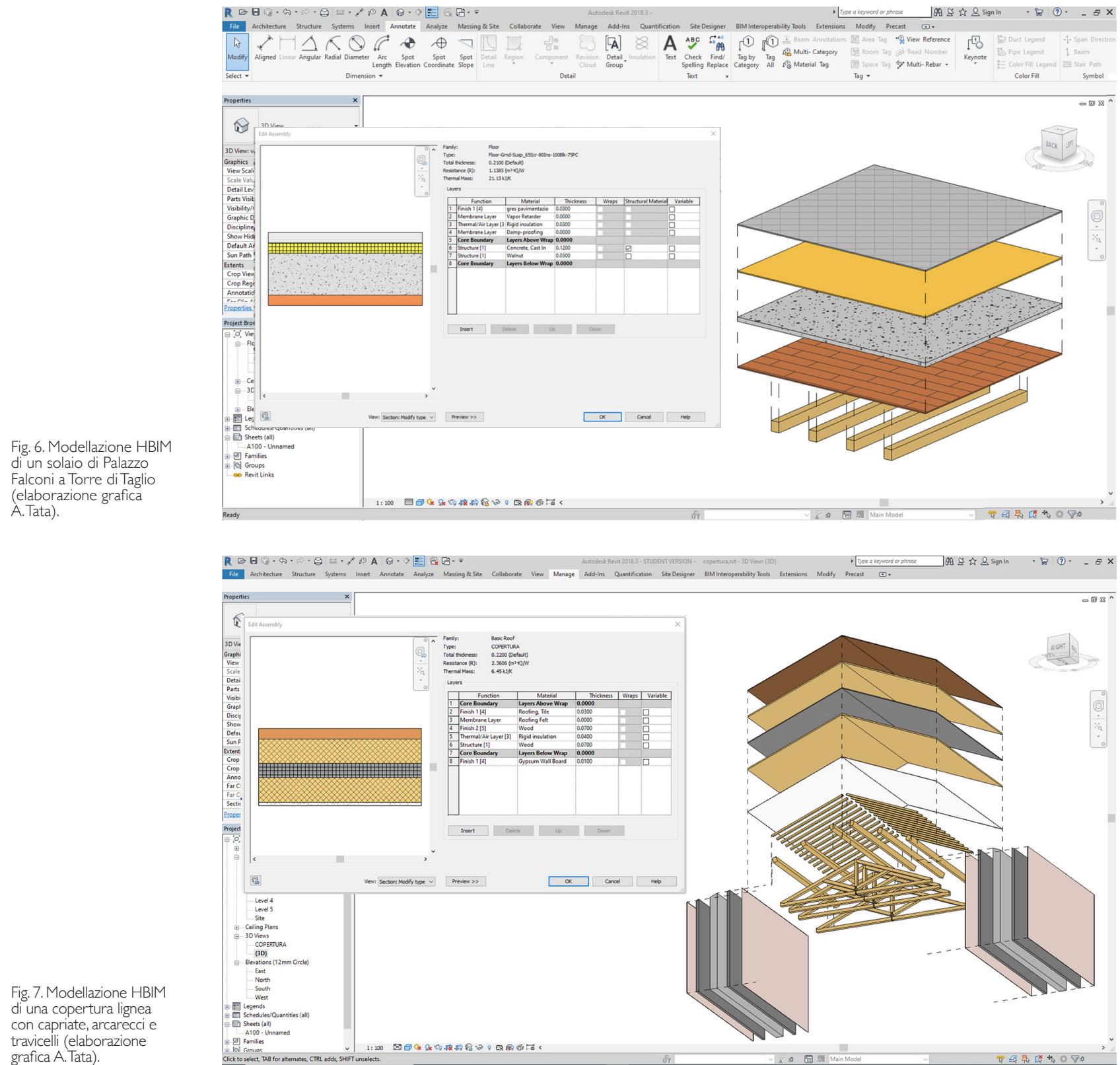


\section{Per un Atlante parametrico dell'apparecchiatura costruttiva storica}

Una importante criticità è posta dall'assenza di librerie parametriche per gli elementi costruttivi storici. Lo sforzo iniziale maggiore (in tempo e lavoro) nella parametrizzazione di nuove famiglie adatte al costruito storico è giustificato dal vantaggio derivante dal loro riutilizzo, non solo all'interno dello stesso modello ma anche in più modelli $[\mathrm{I} I \mathrm{l}$.

Dal punto di vista geometrico infatti, non è sempre produttivo e vantaggioso modellare l'elemento in tutti i suoi singoli dettagli. In una logica di efficienza, tipica del BIM, spesso infatti è più utile un compromesso nella modellazione, con il fine di creare elementi meno dettagliati che però possano far parte di una libreria condivisa e che siano quindi adattabili e riutilizzabili. Ulteriori dettagli possono essere aggiunte tramite documenti di corredo: questo livello di astrazione del modello rinvia a disegni di dettaglio 2D in dwg; documenti e fotografie storiche o elaborati di cantiere sotto forma di pdf o img; video; modelli 3D più dettagliati o parti di essi (ad esempio nurbs o mesh), web links; nuvole di punti derivanti dal rilievo. Queste informazioni possono essere inserite all'interno del database e sono collegabili ai modelli attraverso l'utilizzo e la creazione di parametri.

Pertanto il modello HBIM non vuole essere una virtualizzazione del reale dal punto di vista di una aderenza totale con esso (modello 'as-built' con LoD elevato che rappresenta tridimensionalmente ogni dettaglio dell'oggetto): il suo fine non è quello di sostituirlo, ma di accompagnarlo come strumento euristico utile per la comprensione e per l'analisi interpretativa complessa che si estrinseca attraverso molteplici modalità di comunicazione e diversi livelli di simbolismo e di parametrizzazione.
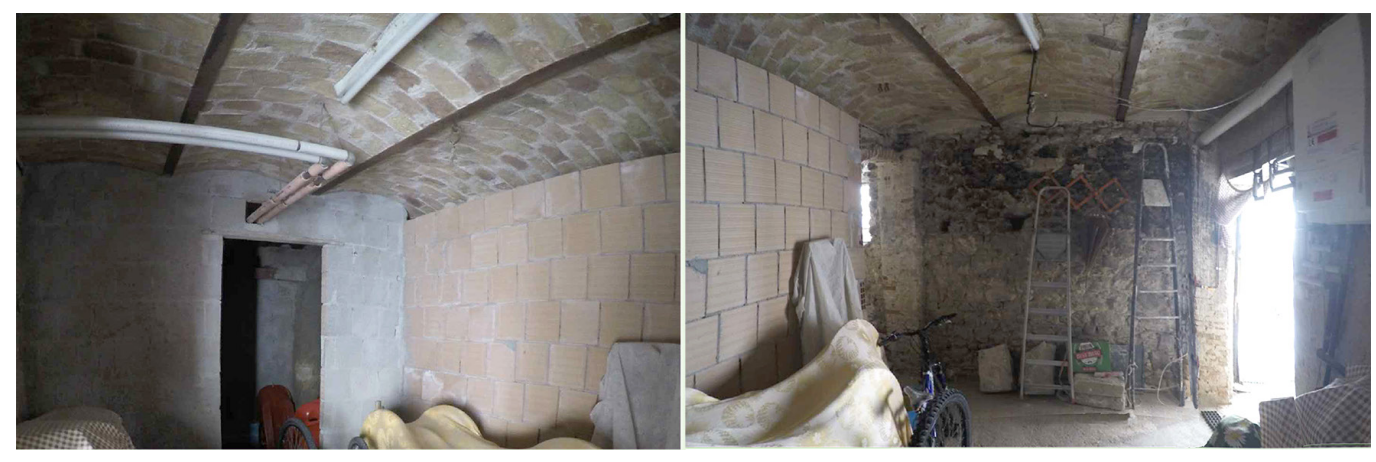

Questo approccio fa il paio con quello riferito ad una conoscenza per 'tipologie' dell'apparecchiatura costruttiva storica, dove però i modelli in quanto parametrici, possiedono la 'capacità' di essere adattati alle singolarità dei manufatti. Certamente l'aderenza non sarà mai totale, ma scientificamente definita con rimandi all'aderenza tra dati e modello interpretativo.

In tal senso la presente ricerca, prendendo le mosse dal rilievo delle apparecchiature costruttive di uno specifico ambito regionale, propone la costruzione di modelli HBIM di elementi costruttivi rilevabili, ma parametricamente definiti negli aspetti geometrici ed informativi - eventualmente anche grazie alle opportunità offerte dalla programmazione visuale [Brusaporci et al. 2019 a; di Luggo et al. 20 I 8] così da poter fungere da 'libreria' per i modelli di altri edifici [Brusaporci et al. 20 I8b].

\section{Conclusioni}

Nell'editoriale del numero della rivista DISEGNARECON intitolato Design for restoration: beyond the survey del 20I5, si legge: "La nuova struttura comunicativa che le innovazioni 
tecnologiche consentono influenza, quindi, questa lettura critica che trae una nuova specificità dalla sua consistenza digitale o, potremmo dire con forse migliore approssimazione, virtuale. L'uso del termine 'oltre' [nel titolo dell'editoriale, n.d.a.] era quindi intenzionalmente indicativo di un potenziale rinnovato aspetto del Rilievo, connesso alla nuova forma di scrittura (digitale invece che su carta) ed ancor più alla potenziata struttura della comunicazione ottenibile in base al tipo di archivio di informazioni che si costruisce (Data Base). [...] La novità reale però, a nostro avviso consiste nel fatto che ora è possibile la costruzione virtuale dell'architettura progettata, la cui potenzialità esplorativa e di controllo sembra superare di gran lunga quella del semplice Disegno proiettivo" [Carbonara e al. 20 I5, pp. E2].Tuttavia, a fronte di visualizzazioni tridimensionali, va tenuto conto che nel passaggio da rappresentazioni $2 \mathrm{D}$ a modelli $3 \mathrm{D}$, i gradi di complessità interpretativa sono moltiplicati, in quanto l'oggetto digitale nello spazio virtuale richiede una sintesi geometrico-dimensionale ed informativa estesa all'intero suo continuum. E come noto, nell'ambito del digitale, proprio il BIM costituisce il primo processo che ontologicamente contempla la gestione e modellazione del sistema costruttivo. Ma un HBIM concettualmente diverso dal BIM in quanto tale, cioè dalla originaria procedura per la realizzazione di nuovi edifici. L'HBIM va in primo luogo riferito ad un processo di conoscenza - sempre aperto e a fondamento al progetto di restauro - riferito ad oggetti digitali caratterizzati da livelli di accuratezza "a macchia di leopardo" relativamente alle informazioni disponibili e alla loro interpretazione [Brusaporci et al. 2019b]. Questo non ne preclude l'utilizzo, quanto - al contrario - ne favorisce un impiego scientifico e rigoroso, anche grazie alle opportunità ormai offerte dalla Programmazione Visuale [12].

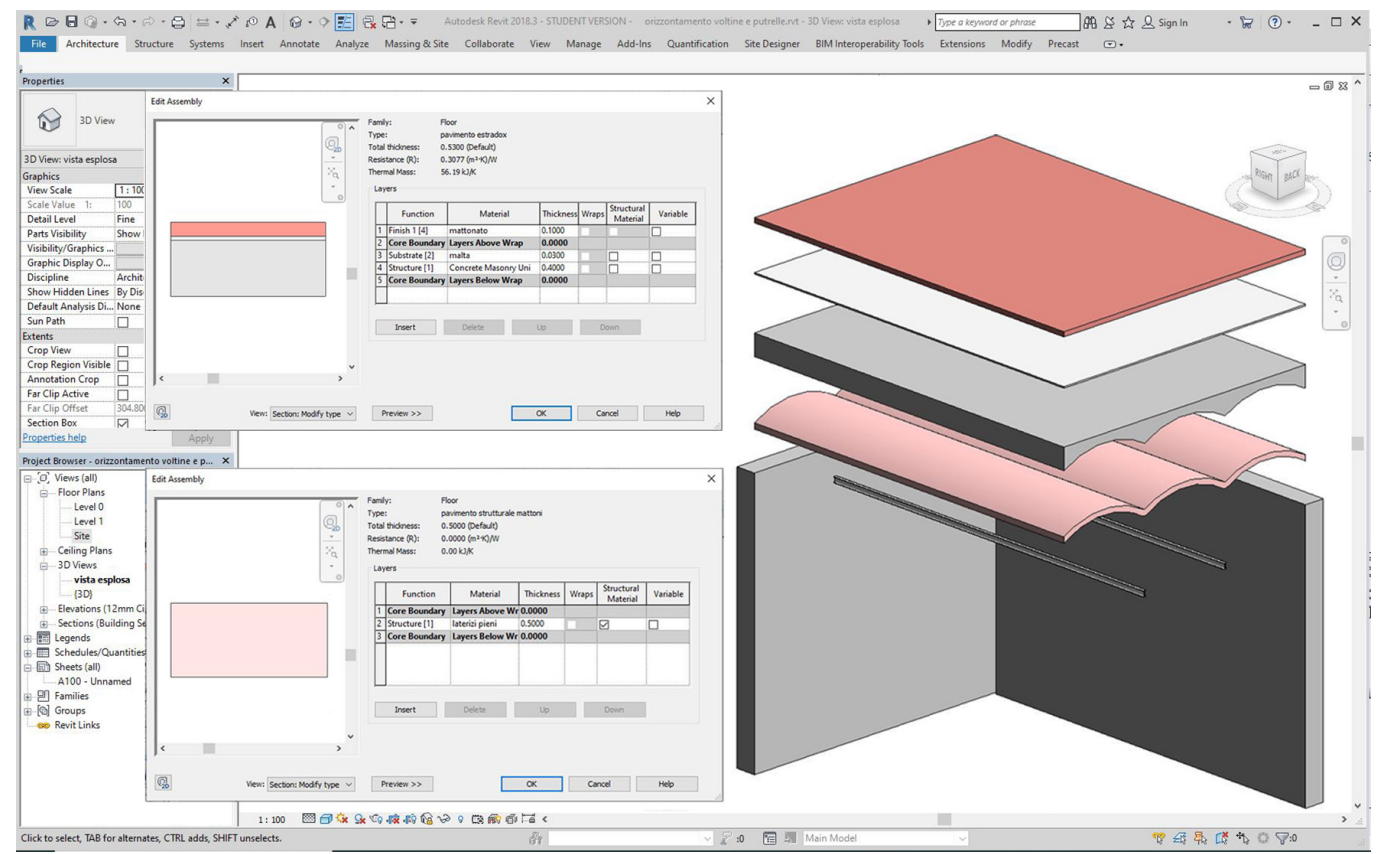

\section{Note}

[I] In informatica il concetto di ontologia è inteso con un'accezione profondamente differente da quella tradizionale del termine in campo filosofico. Nell'ambito della computer science infatti, l'ontologia informatica è stata definita da Tom Gruber come esplicita specificazione di una concettualizzazione.

[2] Tra gli altri si veda: Giuffrè 1993; Giovanetti 1990; Marconi 1997.

[3] Si citano solamente gli Atti del convegno di Udine del 198 I che raccolgono, tra gli altri, i contributi di Sandro Benedetti e di Gianfranco Caniggia, rappresentativi di noti ed importanti filoni di ricerca [AAVV 198I]. 
[4] Data la sterminata pubblicistica, si citano solamente gli studi inerenti il centro Italia, e in particolare sull'Abruzzo, specifica area di sperimentazione della ricerca in oggetto: Zordan et al. 2002; Varagnoli 2000; 2008a.

[5] Oltre ai manuali, si ricordano: Carbonara 1990; Fiorani 2004.

[6] II Manuale di rilevamento architettonico e urbano di Mario Docci e Diego Maestri del 2009 tratta il tema del rilievo dei particolari costruttivi accoppiandolo a quello dei particolari architettonici [p. I I I] e dedicando nel capitolo sulle Metodologie di rilevamento per il restauro uno specifico paragrafo allo studio delle murature. In maniera non dissimile Bertocci e Bini (20 I2), presentano un capitolo sul rilievo degli elementi architettonici storici ed uno sul rilievo "come strumento di interpretazione" dove il "rilievo strutturale" viene inserito nell'ambito del rilievo per il cantiere di restauro. Certamente che lo studio delle geometrie dell'architettura sia correlato a considerazioni sulla natura costruttiva degli elementi stessi è condizione necessaria. Si ricorda anche: Fiorucci, Chiavoni 2003.

[7] Tra i numerosi studi si ricordano: Francovic Parenti 1988; Marta 1989, Marta I991. Nell'ambito del restauro: Fiorani I996. E in particolare per l'area Abruzzese: Brusaporci 2007; Varagnoli 2008b; Varagnoli 2009; Di Nucci 2009.

[8] Le viste spaziali - anche se di progetto - ricorrono sin dalla trattatistica rinascimentale, con pseudo-assonometrie di elementi architettonici e murature, come ad esempio nello Scamozzi. E si ricorda anche la sezione rilievo-progetto di Leonardo Da Vinci per il Duomo di Milano [Codice Atlantico, f.850r], con la rappresentazione dei conci di pietra ammorsati tra loro e la tessitura dei laterizi. Normalmente i disegni di rilievo, anche i più mirabili - come ad esempio le sezioni della Basilica di San Pietro del Ferrabosco ( 1624 ) - per quanto complessi ed accurati, si incentrano sul dettaglio architettonico e non costruttivo. Così anche le rappresentazioni di ruderi di epoca classica, dove i riferimenti ai materiali spesso hanno piuttosto una funzione evocativa, riferibile alla sfera del pittoresco; ma certamente sarà il Piranesi nel XVIII secolo a disegnare i particolari costruttivi delle mura romane.

[9] Dal punto di vista geometrico l'unicità dei componenti edilizi derivante dall'utilizzo di processi di costruzione non industrializzati si contrappone alla logica parametrica e standardizzata tipica degli edifici di nuova costruzione. Molte sperimentazioni trattano dello studio di procedure per la modellazione complessa di queste geometrie [Garagnani 20 I3; Apollonio et al. 2012 Dore, Murphy 20 I3]. Dal punto di vista informativo gli edifici storici sono costituiti da un grande numero di informazioni di varia natura relative a vari temi derivanti dalle ricerche bibliografiche ed archivistiche (documenti e fotografie storiche, dati di monitoraggi, informazioni strutturali, stato di conservazione) [Fai et al. 201 I]. Queste informazioni, non pienamente supportate dai software BIM, per essere inserite all'interno dei modelli devono essere digitalizzate e collegate agli oggetti digitali attraverso un ampliamento del database [Bruno, Roncella 2019].

[10] Oreni et al. 2013, sottolineano come il modello, per essere davvero utile, non deve fermarsi ai soli aspetti geometrici relativi alla superficie degli elementi ma deve estendersi al di là della superficie e deve tenere in considerazione le stratigrafie gli aspetti strutturali e le regole costruttive. Nel loro articolo studiano l'utilizzo del BIM per la costruzione di una libreria degli elementi architettonici (nello specifico volte e solai) che permetta quindi un abaco degli elementi costruttivi locali. León-Robles et al. 20 I 8, utilizzano il BIM per modellare un ponte in pietra definendo ognuno degli elementi che lo costituiscono nei suoi aspetti fisici, le proprietà costruttive e gli aspetti strutturali. II modello del progetto è stato utilizzato per studiare le deformazioni subite dal ponte dalla sua costruzione ad oggi e il BIM è stato impiegato per la gestione e pianificazione delle misure di conservazione da prendere per il futuro.

[I I] La realizzazione di modelli parametrici dell'apparecchiatura costruttiva storica può essere effettuata attraverso l'utilizzo di metodologie differenti a seconda: della tipologia di elemento; dei fini della modellazione; del livello di dettaglio a cui si intende (ed è opportuno) arrivare dal punto di vista geometrico e che è possibile raggiungere dal punto di vista informativo; dal grado di parametrizzazione e riproducibilità che si intende conferire all'oggetto modellato.

Per quanto riguarda le chiusure orizzontali intermedie quindi: nel caso di solaio stratificato con travi in legno sottostanti, è stato creato il pavimento stratificato all'interno della famiglia di sistema 'pavimento' ed è stata inserita una famiglia 'sistema di travi' parametrica sottostante il solaio (fig. 6); nel caso di orizzontamento voltato invece, lo strato strutturale voltato è stato creato utilizzando un solaio strutturale svuotato all'intradosso e all'estradosso da un vuoto parametrico e, al di sopra di quest'ultimo, è stato inserito un solaio architettonico stratificato svuotato, in corrispondenza della volta parametrica, da un ulteriore vuoto parametrico (fig. 9). Per quanto riguarda il solaio di copertura con arcarecci, travi e capriate in legno, è stato creato un "tetto" stratificato che è stato completato al di sotto da due sistemi di travi perpendicolari tra di loro con travi a sezione differente. e da capriate parametriche (fig. 7).

[12] II presente contributo è stato concepito congiuntamente dagli autori. Tuttavia i paragrafi possono essere attributi come segue: S. Brusaporci è autore dei paragrafi: Introduzione e Rappresentare l'apparecchiatura costruttiva storica; A. Tata dei paragrafi: L'apparecchiatura costruttiva storica nei processi HBIM, e Per un Atlante parametrico dell'apparecchiatura costruttiva storica; M. Centofanti delle Conclusioni.

\section{Riferimenti bibliografici}

AAVV ( | 98I). II recupero dei vecchi centri. Gli aspetti teorici. I modi d'intervento. Atti del Convegno Internazionale di Studi, Udine 22-24 maggio 1981. Udine: Università degli Studi di Udine.

Apollonio Fabrizio Ivan, Gaiani Marco, Zheng Sun (2012). BIM-based modeling and data enrichment of classical architectural buildings. In SCIRES-it, 2, 2012 , 2, pp. 4I-62.

Baik Ahmad, Alitany Ayman, Boehm Jan, Robson Stuart (20|4). Jeddah Historical Building Information Modelling “JHBIM" Object Library. In ISPRS Ann. Photogramm. Remote Sens. Spatial Inf. Sci., II-5, 20 I 4, pp. 4 I -47.

Bertocci Stefano, Bini Marco (2012). Manuale di rilievo architettonico e urbano. Novara: Città Studi.

Bianchini Carlo, Nicastro Saverio (2018). La definizione del Level of Reliability: un contributo alla trasparenza dei processi di Historic-BIM. In EmplerTommaso, Valenti Graziano Mario (a cura di). 3D Modeling \& BIM Nuove Frontiere. Roma: dei Tipografia del genio civile, pp. 208-225.

Bruno Nazarena, Roncella Riccardo (2019). HBIM for conservation: A new proposal for information modelling. In Remote Sensing, II, 2019, 15, pp. I75।-1774. 
Brusaporci Stefano (2007). Le murature dell'architettura del versante meridionale del Gran Sasso (secc. XI-XIV). Roma: Gangemi editore.

Brusaporci Stefano (2017). The Importance of Being Honest: Issues of Transparency in Digital Visualization of Architectural Heritage. In Ippolito Alfonso. Handbook of Research on Emerging Technologies for Architectural and Archaeological Heritage. Hershey, PA: IGI Global, pp. 66-93.

Brusaporci Stefano, Maiezza Pamela,Tata Alessandra (20 I 8a). A Framework for Architectural Heritage HBIM Semantization and Development. In The International Archives of the Photogrammetry, Remote Sensing and Spatial Information Sciences. 20 I 8. XLII-2, pp. $179-184$.

Brusaporci Stefano, Maiezza Pamela, Tata Alessandra (2018b). Computational Design for As-Built Modeling of Architectural Heritage in HBIM processes. In 20 I 8 IEEE International Conference of Metrology forArchaeology and Cultural Heritage, MetroArchaeo Proceedings. Cassino 22-24 October 2018, pp.194-199.

Brusaporci Stefano, Maiezza Pamela,Tata Alessandra (2019a). Prime riflessioni sulla rappresentazione e parametrizzazione HBIM dell'apparecchiatura costruttiva storica. In Empler Tommaso, Fusinetti Alexandra (a cura di). 3D Modeling \& BIM Modelli e soluzioni per la digitalizzazione. Roma: dei Tipografia del genio civile, pp. I82-197.

Brusaporci Stefano, Maiezza Pamela, Tata Alessandra (2019b). Trasparenza e affidabilità dei modelli HBIM. Transparency and reliability of HBIM models. In Papa Lia Maria, D'Agostino Pierpaolo (a cura di). BIM Views: Esperienze e scenari. Fisciano, SA: CUA, pp. $125-140$.

Carbonara Giovanni, Centofanti Mario, Mingucci Roberto (20 I 5). Disegno per il restauro: oltre il rilievo. In Disegnarecon, n. I4, 20I5, 8, pp. E2.

Carbonara Giovanni (1990). Restauro dei Monumenti. Guida agli elaborati grafici. Napoli: Liguori.

Carbonara Giovanni (1989). Disegnare per il restauro. In Disegnare. Idee Immagini, 0, 1989, pp. 85-94.

Centofanti Mario (20 I0). Della natura del modello architettonico. In Brusaporci Stefano (a cura di). Sistemi informativi integrati per La tutela la conservazione e la valorizzazione del patrimonio architettonico e urbano. Roma: Gangemi, pp. 43-54.

Cetra Valentina (20 I 5). Progetto di restauro di un aggregato nel centro storico di Castel del Monte (AQ). Tesi di Laurea in Ingegneria Edile-Architettura, Università degli Studi dell'Aquila. A.A. 20 I 4/20 I 5. Relatori: Centofanti Mario, Brusaporci Stefano. Correlatore: Di Fabio Franco.

Curti Mariangela (20I5). Rilievo e restauro del palazzo baronale Falconi in Torre di Taglio (RI). Tesi di Laurea in Ingegneria EdileArchitettura, Università degli Studi dell'Aquila. A.A. 20 I 4/20 15. Relatore: Centofanti Mario. Correlatore: Brusaporci Stefano.

Di Luggo Antonella, Scandurra Simona, Pulcrano Margherita et al. (2018). Sistemi voltati nei processi cloud to BIM. In Empler Tommaso, Valenti Graziano Mario (a cura di). 3D Modeling \& BIM Nuove Frontiere. Roma: dei Tipografia del genio civile. vol 2, pp. $162-177$.

Di Nucci Anna (2009). L'arte di costruire in Abruzzo. Tecniche murarie nel territorio della Diocesi di Valva e Sulmona. Roma: Gangemi editore.

Docci Mario, Maestri Diego (2009). Manuale di rilevamento architettonico e urbano. Bari: Laterza, pp. 374.

Dore Conor, Murphy Maurice (2013). Semi-Automatic Modelling of Building Façades with Shape Grammars Using Historic Building Information Modelling. In International Archives of the Photogrammetry, Remote Sensing and Spatial Information Sciences, XL-5/WI, pp. 57-64.

Fai Stephen, Graham Katie, Duckworth Todd et al. (201 I). Building Information Modelling and Heritage Documentation. In Fai Stephen, Graham Katie et al. (eds). Conference Proceedings: XXIII CIPA International Symposium. Praga I2-16 September 201 I. Praga: CIPA Heritage Documentation.

Fiorani Donatella (1996). Tecniche costruttive murarie medievali. Roma: L'Erma di Bretschneider.

Fiorani Donatella (2004). Restauro Architettonico e strumento informatico. Guida agli elaborati grafici. Napoli: Liguori.

Fiorucci Tiziana, Chiavoni Emanuela (2004). Gli strumenti di conoscenza per il progetto di restauro. Roma: Gangemi editore.

Francovic Riccardo, Parenti Roberto (1988). Archeologia e Restauro dei monumenti. Firenze: L'insegna del Giglio.

Garagnani Simone (2013). Building Information Modeling a real world knowledge. A methodological approach to accurate semantic documentation for the built environment. In DigitalHeritage 20 I 3. Marsiglia: IEEE, pp. 489-496.

Giovanetti Francesco (1990). Manuale del recupero di Città di Castello. Città di Castello: Comune di Città di Castello.

Giuffrè Antonino (1993). Sicurezza e conservazione dei centri storici: il caso Ortigia: codice di pratica per gli interventi antisismici nel centro storico. Roma-Bari: Laterza.

Graziani Enrica M. L. (20 I5). Rilievo e Restauro di Palazzo Loddi (XVII sec.) L'Aquila. Tesi di Laurea in Ingegneria Edile-Architettura, Università degli Studi dell'Aquila. A.A. 2014/2015. Relatore: Centofanti Mario. Correlatori: Brusaporci Stefano, Quaresima Raimondo.

León-Robles Carlos A., Reinoso-Gordo Juan F., González-Quiñones Juan J. (2018). Heritage Building Information Modeling (H-BIM) Applied to A Stone Bridge. In ISPRS Int. J. Geo-Information, 20 19, 8, p. 121 - 135.

Logothetis S., Delinasiou A., Stylianidis E. (20I5). Building Information Modelling for Cultural Heritage: A review. In ISPRS Ann. Photogramm. Remote Sens. Spatial Inf. Sci., II-5/W3, 2015, pp. I77- 183. 
Maiezza Pamela (2019). As-Built reliability in architectural HBIM modeling. In The International Archives of the Photogrammetry, Remote Sensing and Spatial Information Sciences, 20I9, XLII-2M9, pp. 46I-466.

Marconi Paolo (1997). Manuale Del Recupero Del Centro Storico Di Palermo. Palermo: Flaccovio, pp. 392.

Marta Roberto (1989). Tecnica Costruttiva a Roma nel medioevo. Roma: Kappa, pp. 160.

Marta Roberto (199I). Tecnica Costruttiva Romana. Roma: Kappa.

Quattrini Ramona, Pierdicca Roberto, Morbidoni Christian (2017). Knowledge-based data enrichment for HBIM: Exploring high-quality models using the semantic-web. In Journal of Cultural Heritage, 28, 20 I 7, pp. I29- I 39.

Murphy Maurice, McGovern Eugene, Pavia Sara (2009). Historic building information modelling (HBIM). In Structural Survey, 27, 4, pp. 3II-327.

Oreni Daniela, Brumana Raffaella, Georgopoulos Andreas et al. (20I3). HBIM for Conservation and Management of Built Heritage: Towards a Library of Vaults and Wooden Bean Floors. In ISPRS Annals of the Photogrammetry, Remote Sensing and Spatial Information Sciences, Volume II-5/WI, 2013, pp. 2I5-22I.

Varagnoli Claudio (2000). Materiali per un atlante della costruzione storica in Abruzzo. In Contributi, 7, 2000.

Varagnoli Claudio (2008a). La costruzione tradizionale in Abruzzo. Fonti materiali e tecniche costruttive dalla fine del Medioevo all'Ottocento. Roma: Gangemi editore.

Varagnoli Claudio (2008b). Terre murate. Ricerche sul patrimonio architettonico in Abruzzo e Molise. Roma: Gangemi editore.

Varagnoli Claudio (2009). Muri parlanti. Prospettive per l'analisi e la conservazione dell'edilizia tradizionale. Firenze: Alinea.

Zordan Luigi, Bellicoso Alessandra, De Berardinis Pierluigi et al. (2002). Le tradizioni del costruire della casa in pietra: materiali, tecniche, modelli e sperimentazioni. L'Aquila: GTE.

\section{Autori}

Stefano Brusaporci, Università degli Studi dell'Aquila, stefano.brusaporci@univaq.it.

Alessandra Tata, Università degli Studi dell'Aquila, alessandra.tata@graduate.univaq.it.

Mario Centofanti, Università degli Studi dell'Aquila, mario.centofanti@univaq.it.

Per citare questo capitolo: Brusaporci Stefano, Tata Alessandra, Centofanti Mario (2020). Tecnologie avanzate per la rappresentazione dell'apparecchiatura costruttiva storica: HBIM e il rinnovarsi di una istanza/Advanced technologies for the representation of historical construction systems: HBIM and the renewal of an instance/ln Arena A., Arena M., Brandolino R.G., Colistra D., Ginex G., Mediati D., Nucifora S., Raffa P. (a cura di). Connettere. Un disegno per annodare e tessere. Atti del $42^{\circ}$ Convegno Internazionale dei Docenti delle Discipline della Rappresentazione/ Connecting. Drawing for weaving relationships. Proceedings of the 42th International Conference of Representation Disciplines Teachers. Milano: FrancoAngeli, pp. 1778-1799. 


\title{
Advanced Technologies for the Representation of Historical Construction Systems: HBIM and the Renewal of an Instance
}

\author{
Stefano Brusaporci \\ Alessandra Tata \\ Mario Centofanti
}

Abstract

The topic of the survey and representation of historical constructive systems is essential in the historical-critical knowledge process of architectural artefacts. In particular, the knowledge of construction systems in some cases rises from a direct interrogation, but often it is the result of indirect investigations or information. Moreover, the high level of iconicity of the representations combines precise knowledge with elements taken from similar case studies. The HBIM procedures, ontologically I developed for an informative management of constructive systems, renews the modelling issues, combining in the information management process both 3D objects and traditional two-dimensional drawings, according to a process where instances of "transparency" of sources and "reliability" of information play a leading role. First, the paper focuses on the state of the art, then it analyses the possibilities offered by HBIM, with particular reference to the opportunities given by the use of parametric objects for the modelling of historical building elements.

Keywords

architectural surveying, constructive systems, HBIM, 3D modelling, vpl.

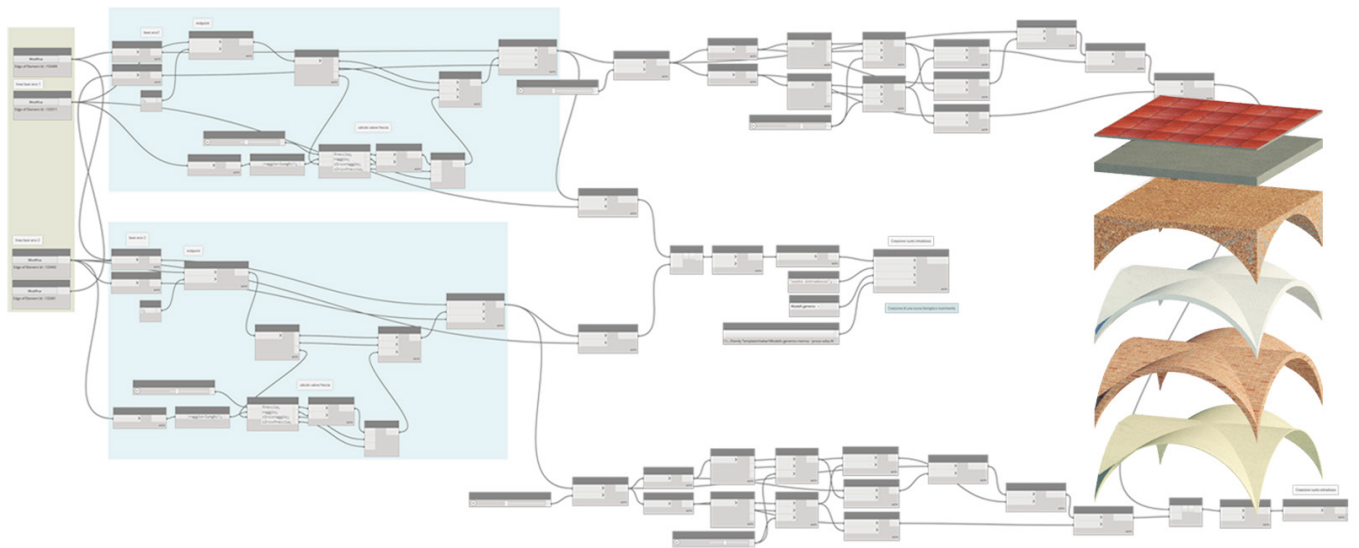




\section{Introduction}

In the Italian tradition, in particular at the end of the Eighties, disciplines interested in "built heritage" focused on the issues of surveying the architectural systems. In particular, the experiences of the so called 'Handbooks', 'Codes of Practice', and 'Atlas' [2] refer to a research line dedicated to historical centres [3], carried out with specific attention to smaller towns and traditional architecture [4]. This attention has shifted the focus from the monumental -albeit related to its context- to an inclusive approach, that correlates the dimensions of the natural and the anthropic, at different scales. This area includes the expressions of constructive culture, according to a sphere where the artefact, intended as a testimony of human doing, arises as a main document, often even in the absence of significant documentary references.

The study, survey and representation of historical construction systems find specific insights especially in the fields of Architectural Restoration [5], Drawing [6], and Archaeology, with particular reference to building techniques, both ancient and medieval [7]. If in the last decade this theme seems rather relegated to specific local contributions. On the other hand, $\mathrm{HBIM}$ procedures require a new reflection, because, in the field of digital architectural technologies, first they are semantically based on information management and digital modelling of construction systems.

\section{Representing historical constructive systems}

The issue of historical construction system surveying is appropriately addressed by the main architectural survey handbooks [Docci, Maestri 2009; Bini, Bertocci 20 I2]. The representation is traditionally conducted with plans and sections at various scales, but also with cross-sections and exploded axonometric views, useful to illustrate the articulation of the different components and their relationships [8].

Carbonara writes in the first issue of the journal Disegnare Idee Immagini: "It is therefore a question of historically understanding and critically drawing and surveying both in great
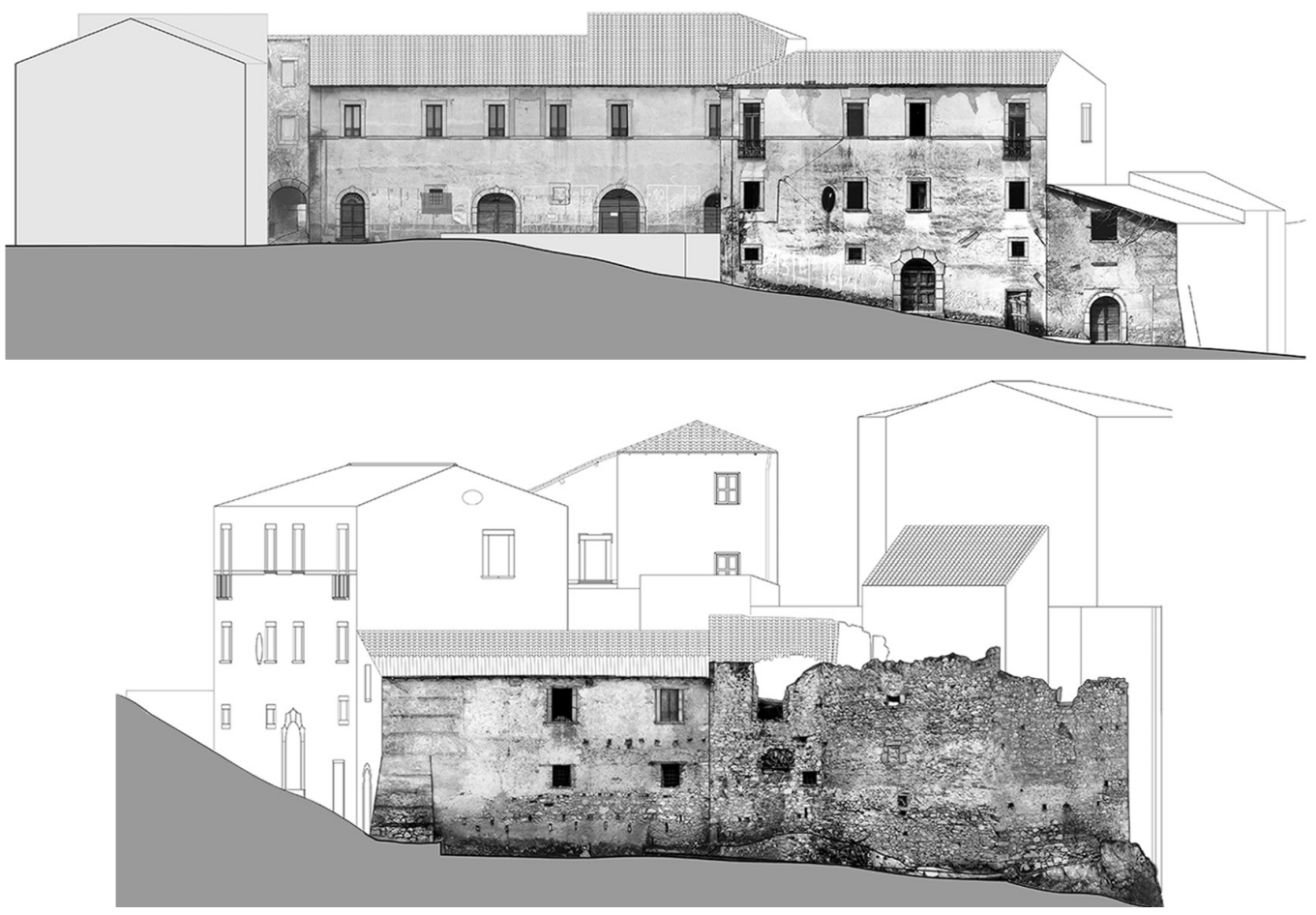
and detail, of seeing and representing in depth [...] first made with the help of a careful inspection, of assiduous and personal attendance of the artefact. Of an anticipation of historical-critical diagnostic analysis implemented with the sight and the touch, with relatively simple means but with sensitivity and experience that remembers, calling into question the usual comparison of medicine with restoration, the good practice of the old doctor's family" [Carbonara 1989, pp 85]. Centofanti defines it "anatomical survey" [Centofanti 20I0, pp. 43-54], which develops the theme of the interpretative model, with specific reference to the issue of the study of construction system, also in three-dimensional and digital dimensions.

The knowledge of the construction system is in most cases an indirect experience of a complex system that is hidden behind the surface. It can sometimes be direct and complete (at least for some parts), elsewhere discrete for texts and introspections, or derive from archival documents, finally, it can be a reconstructive hypothesis, conducted on the basis of the scholar's experience and culture, or suggested by studies or handbooks. Only in the construction site phase, it can find an epiphany. Therefore, when dealing with the representation of the construction system of the building, knowledge and representation is largely based on the use of symbols or often on the representation -and adaptation- of typologically similar systems, surveyed elsewhere. For example, in the sectional drawing of a masonry, it can be represented with reference to a 'system' to highlight its peculiarities (type of elements, maximum, average and minimum dimensions, installation method). It is a game of references between a drawing of likelihood in the representation of the components, and interpretation of the construction system. Therefore, the 'fragmentation' of direct knowledge leads to graphic models strongly outlined in details, but which only partly derive from direct measurement, and which aim to describe the significant characteristics of what is represented, in a game between specificity of the product and construction type.

This aspect could usefully interface with the BIM approach for parameterized objects. In any case, issues relating to the 'Transparency' (ie the declaration of the sources and processes of knowledge) and the 'Reliability' (ie the geometric and informative interpretative limits) of the digital construction components are essential [Brusaporci 2017; Bianchini, Nicastro 20l8; Maiezza 2019].

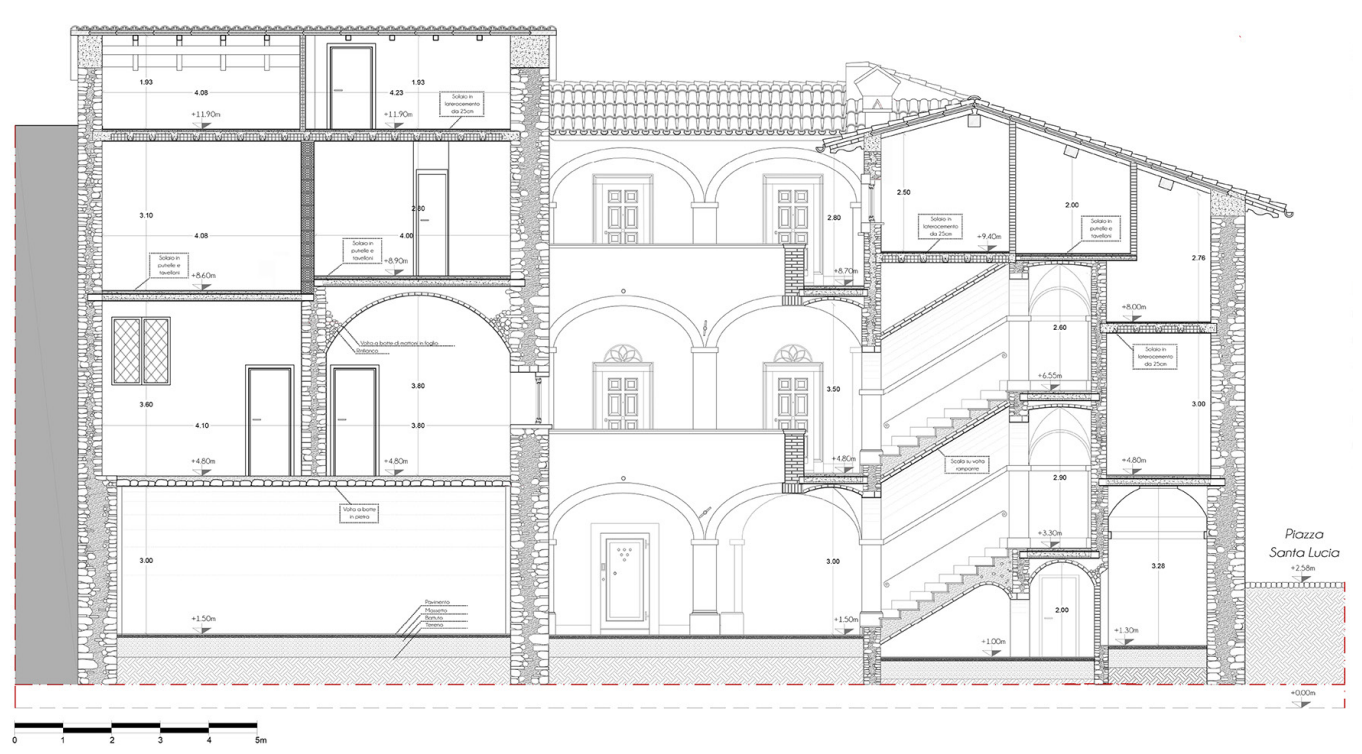


Fig. 3. Surveying of the carpentry of the roofs of a building in the historic centre of Castel del Monte (graphic elaboration by V. Cetra).

Fig. 4. Surveying of the constructive system of Palazzo Falconi in Torre di Taglio (graphic elaboration by M. Curti).
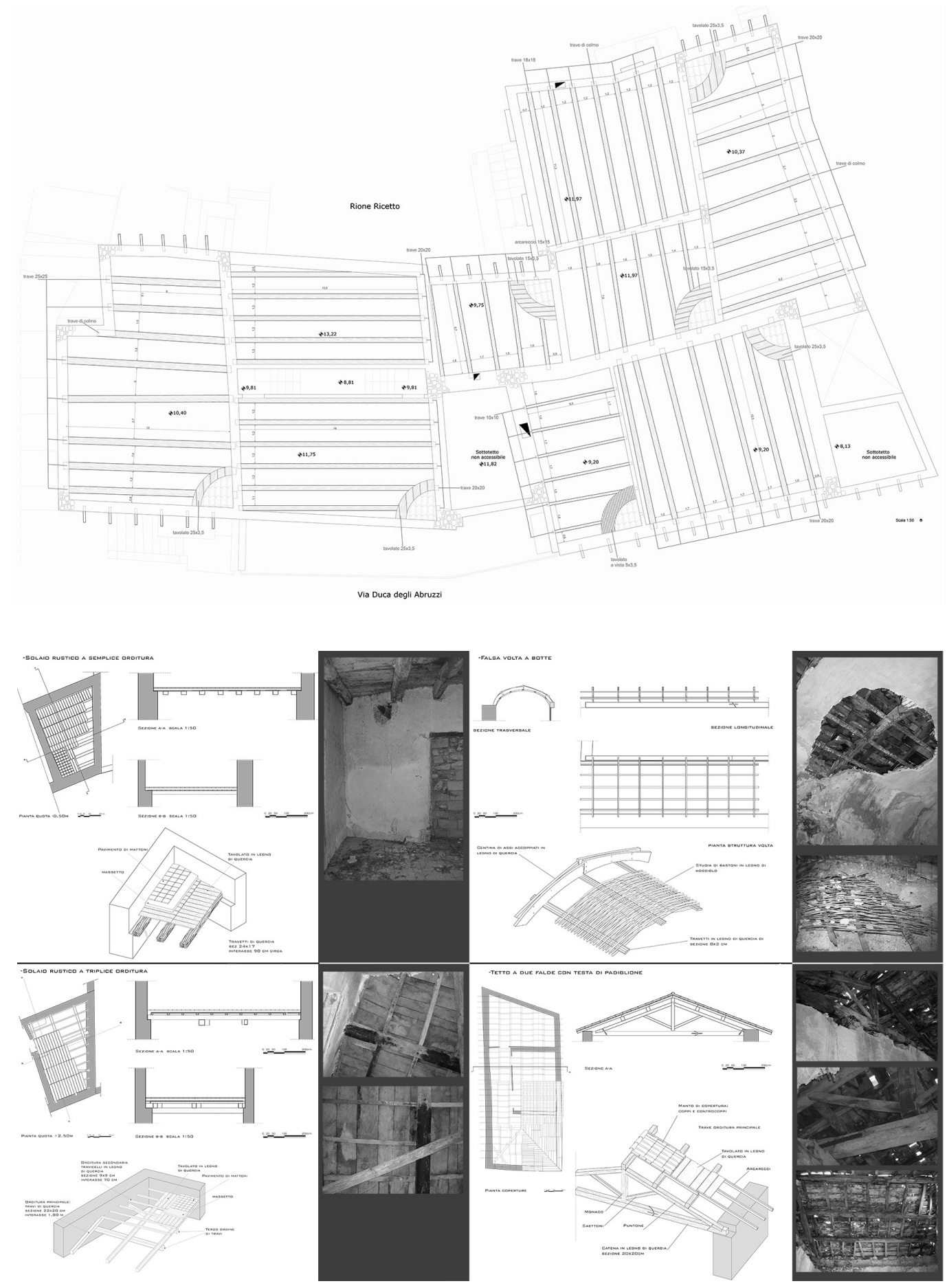


\section{Historical Construction System in HBIM Processes}

BIM is a process for managing information related to buildings. The database refers to a 3D model, semantically defined in relation to the construction equipment. With specific reference to the most recent line of development that places the so-called LOINs (Level of Information Need, that is the required information levels) at the centre of the process, it is clear that the model does not stands as a synthetic clone of reality (both in the project and in the survey), but arises as an interpretative model, where traditional LoD (Levels of development) can, depending on the circumstances and purposes, present different definitions, articulations and characterizations. If necessary, in fact, the model can be enriched with traditional two-dimensional drawings or alphanumeric data.

Despite the close relationship between the geometric and informative aspects within the BIM process, today most of the research lines relating to the use of HBIM are aimed at either the geometric-dimensional representation of historical building components or the built heritage documentation [9]. Within these experiments, however, there are only a few studies that deal with the theme of the representation of the construction equipment of historic buildings [10].
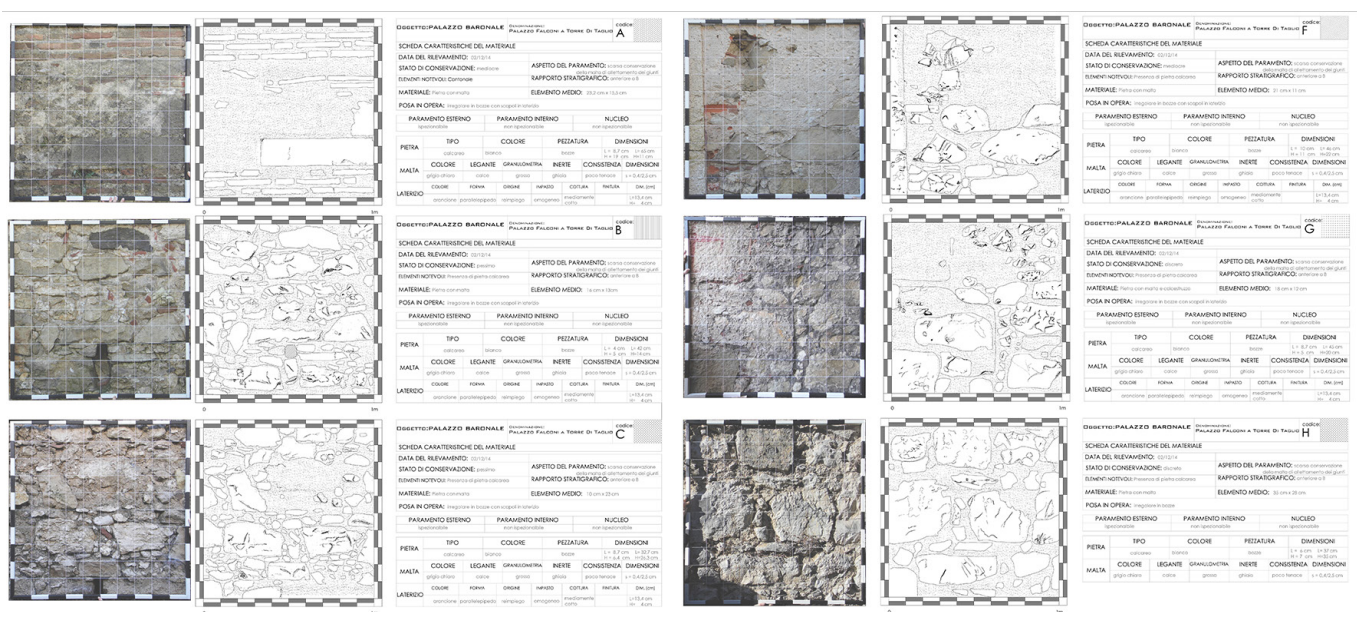

The importance of the information aspect within the BIM logic is also underlined by the current Italian and foreign regulations. In fact, UNI I I 337: 20 I7, in accordance with the British PAS I 192-2 system of 20 13, splits the level of development of LoD objects into: LoG, level of development of objects- geometric attributes, and Lol, level of development of objectsinformative attributes.

The knowledge of the construction equipment is mostly fragmentary, generally not exhaustive, deriving from direct or indirect experiences. The HBIM model therefore consists of elements with non-uniform LoD in which the modeling of the components will depend on the available information and will be the result of a critical process in which the modeler, in case of lack of certain information, will also make his experience available for put forward interpretative hypotheses. Therefore, the operational choices must be described in relation to the basic knowledge documentation, according to the known principles of 'Transparency' and 'Reliability' of the model objects [Bianchini. Nicastro 20I8; Brusaporci et al. 20 I8]. The importance assumed by these concepts is confirmed by UNI which defines LoD as measured by the "nature, quantity, quality and stability of data and information" associated with each digital object; therefore, with progressing from one LoD to the next is associated an increase both in the quantity of information and in its quality, in the sense of reliability and data consolidation. 
Fig. 6. HBIM modelling of a slab in Palazzo Falconi in Torre di Taglio (graphic elaboration by A. Tata).
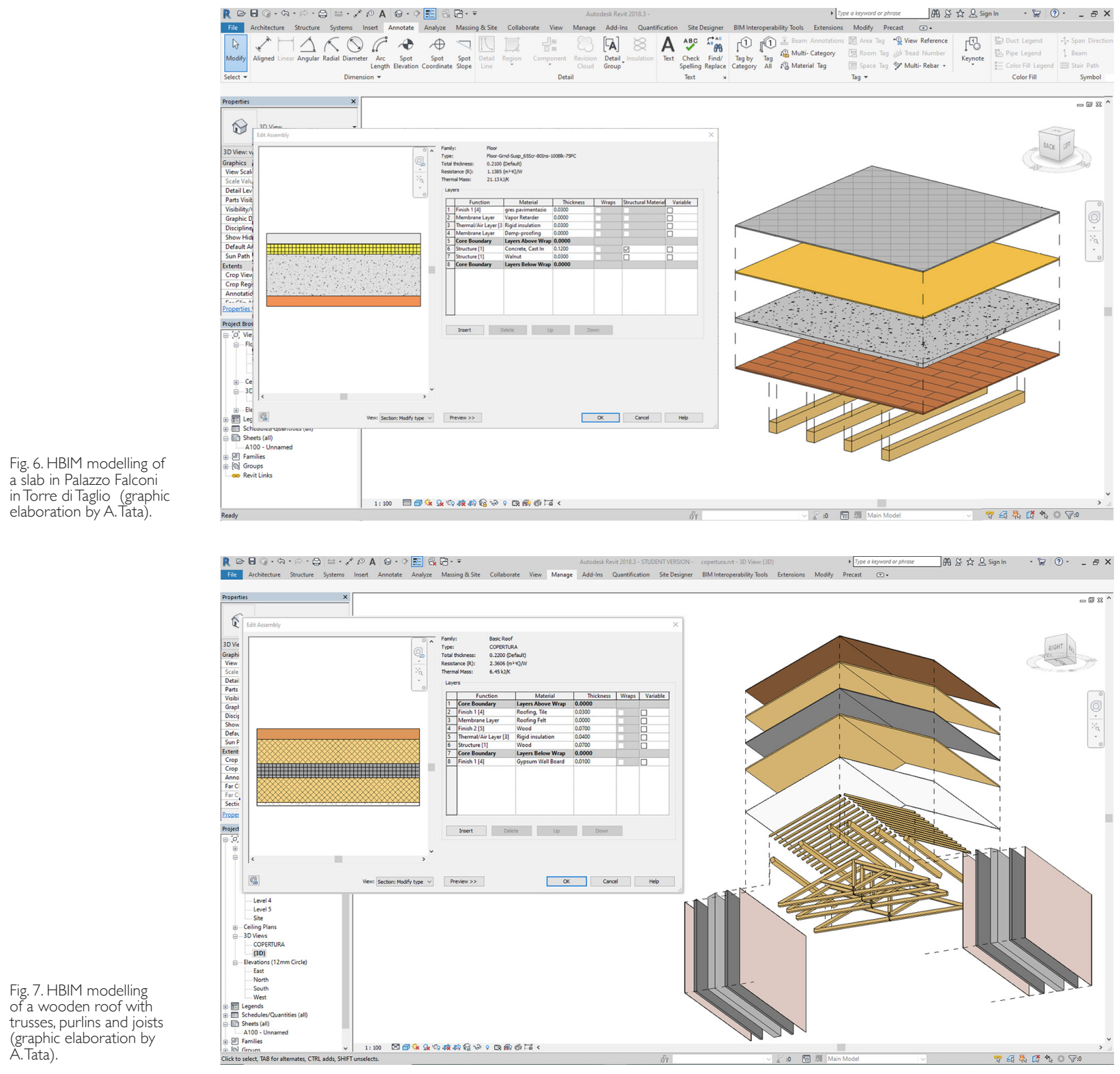


\section{For a parametric Atlas of Historical Construction System}

An important critical issue is the absence of parametric libraries for historical building elements. The greater initial effort (in time and work) in the parameterization of new families suitable for the historical building is justified by the advantage deriving from their reuse, not only within the same model but also in multiple models [ $\mathrm{l}$ l].

From a geometric point of view, in fact, it is not always productive and advantageous to model the element in all its individual details. In a logic of efficiency, typical of BIM, a compromise in modeling is often more useful, with the aim of creating less detailed elements which, however, can be part of a shared library and which are therefore adaptable and reusable. Further details can be added through accompanying documents: this level of model abstraction refers to 2D detail drawings in dwg; historical documents and photographs or construction documents in the form of pdf, or img; video; more detailed 3D models or parts of them (for example nurbs or mesh), web links; point clouds deriving from the survey. This information can be entered within the database and can be connected to the models through the use and creation of parameters.

Therefore the HBIM model does not want to be a virtualization of reality from the point of view of total adherence with it ('as-built' model with high LoD that represents every detail of the object in three dimensions): its purpose is not to replace it, but to accompany it as a useful heuristic tool for understanding and for complex interpretative analysis that is expressed through multiple communication modes and different levels of symbolism and parameterization.

Fig. 8. Brick vaults of a building in the historic centre of Castel del

Monte (AQ), (photo by V. Cetra).
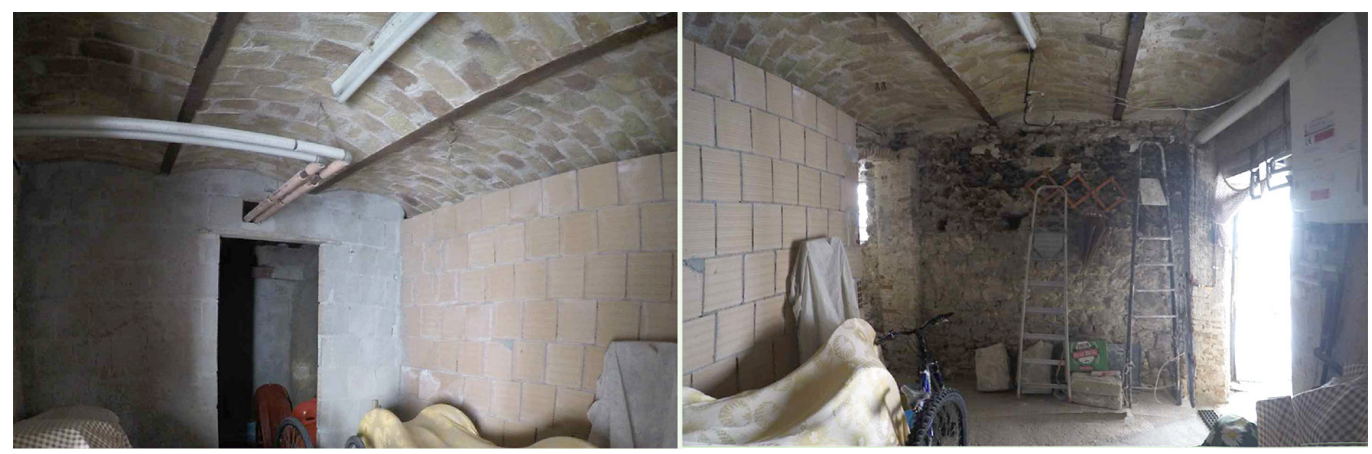

This approach goes hand in hand with that referred to a knowledge for 'types' of historical construction equipment, where however the models as parametric, possess the 'ability' to be adapted to the singularities of the artefacts. Certainly the adherence will never be total, but scientifically defined with references to the adherence between data and interpretative model.

In this sense, the present research, starting from the survey of the construction equipment of a specific regional area, proposes the HBIM modeling of surveyable construction elements, but parametrically defined in the geometric and informative aspects - possibly also thanks to the opportunities offered by visual programming [Brusaporci et al. 20 I 9a; di Luggo et al. 20 I 8] so that it can act as a 'library' for other buildings models [Brusaporci et al. 20 I 8b].

\section{Conclusions}

From the Editorial of the issue of the journal DISEGNARECON titled Design for restoration: beyond the survey, 2015:"The new communication structure of technological innovations influences therefore, this critical reading that draws a new specificity from its digital consisten- 
cy or, we could say, with perhaps better approximation, virtual. The wording 'beyond' in the title is intentionally indicative of a potential renewed aspect of the Survey, connected to the new form of writing (digital instead of paper) and even more to the enhanced communication structure obtainable on the basis of the type of information archive (Data Base). [...] The real news, however, in our opinion consists in the fact that virtual construction of the designed architecture is now possible, whose exploratory and control potential seems to far exceed that of simple projective Drawing" [Carbonara e al. 20I5, pp. E2]. However, in the transition from 2D representations to 3D models, the degrees of the interpretative complexity are multiplied since the digital object in the virtual space requires a geometric-dimensional and information synthesis extended to its entire continuum. And as is known, in the digital field, BIM is the first process that ontologically contemplates the management and modelling of the construction system. But a HBIM is conceptually different from BIM as such, that is, from the original procedure for the construction of new buildings. The HBIM is primarily a process of knowledge, referring to digital objects characterized by levels of 'spotted' accuracy in relation to the information available and their interpretation [Brusaporci et al. 2019b]. This does not preclude its use, as -on the contrary- it favours a scientific and rigorous use, also thanks to the opportunities offered by Visual Programming [12].

Fig. 9. HBIM modelling of vaults made by iron beam and bricks of a building in Castel del Monte (graphic elaboration by A.Tata).

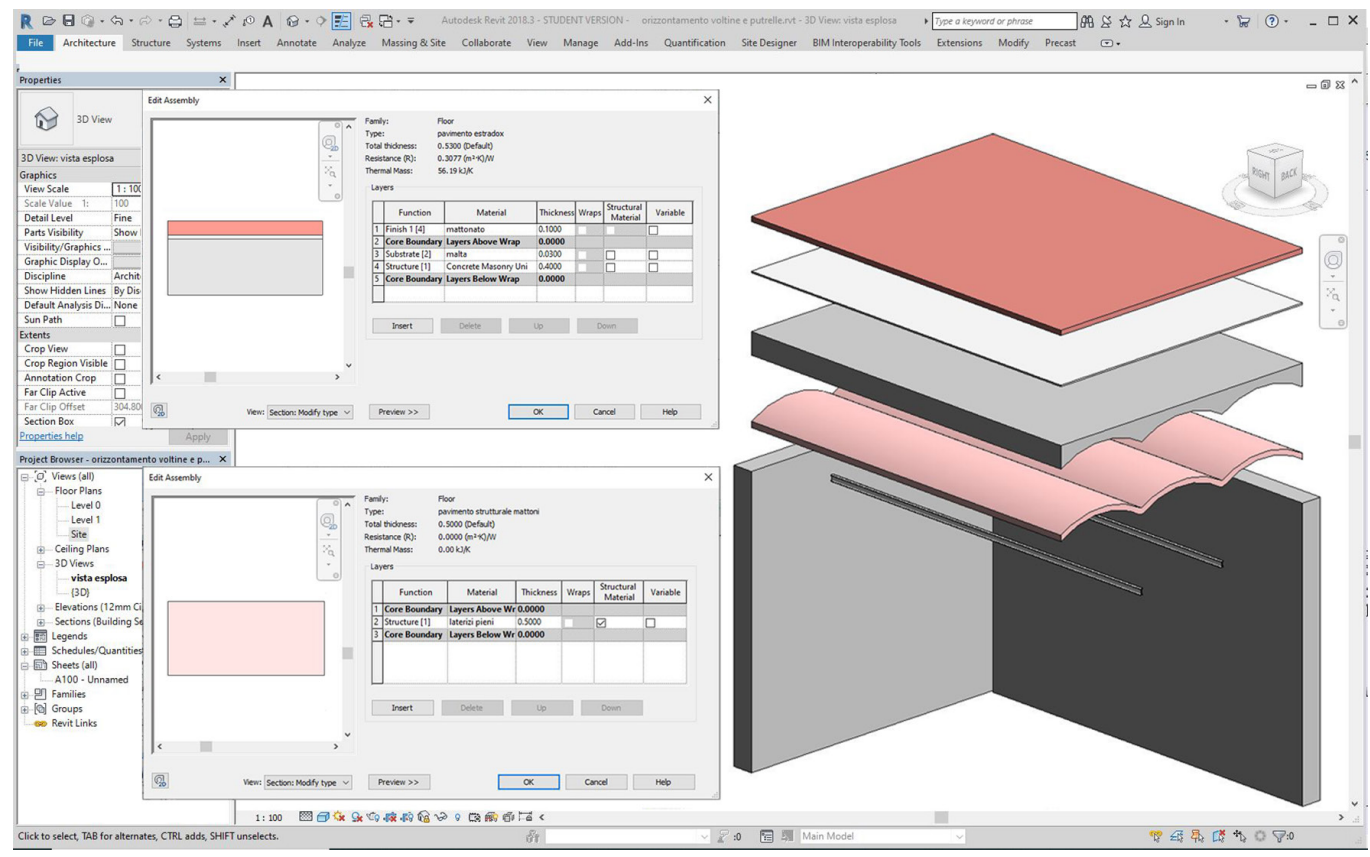

\section{Notes}

[I] In computer science, the concept of ontology is different from the traditional one in the philosophical field: IT ontology has been defined by Tom Gruber as an explicit specification of a conceptualization.

[2] In particular: Giuffrè 1993; Giovanetti 1990; Marconi 1997.

[3] We only cite the Proceedings of the Conference held in Udine in 1981, where there are the papers of Sandro Benedetti and Gianfranco Caniggia, representative of well known and important lines of research [AAVV I981].

[4] Considering the large amount of publications, we cited only the studies related to central Italy, and in particular to Abruzzo, the specific area of research of this experimentation: Zordan et al. 2002; Varagnoli 2000, 2008a.

[5] We remember: Carbonara 1990; Fiorani 2004.

[6] The Handbook by Docci and Maestri on the urban and architectural survey, 2009 deals with the surveying of constructive details, combining them with that of the architectural details [Docci, Maestri 2009 p. I 2 I]; in the chapter on Surveying method- 
ologies for restoration, there is a specific paragraph on masonry study. In a similar way: Bertocci, Bini 2012 present a chapter on the survey of historical architectural elements and another on the survey as 'interpretation tool' where the structural survey is presented in relation to the survey for the restoration yard. Certainly, the study of the geometries of architecture has to be related to considerations on the constructive nature of the elements themselves. We also remember: Fiorucci, Chiavoni 2003.

[7] Among the numerous studies, we rememeber: Francovic, Parenti 1988; Marta 1989; Marta I99I. In the field of restoration: Fiorani 1996. For the Abruzzo Region: Brusaporci 2007; Varagnoli 2008b; 2009; Di Nucci 2009.

[8] Spatial views - even if project-based - recur since the Renaissance treatises, with pseudo-axonometries of architectural elements and walls, as for example in Scamozzi. And we also remember the survey-project section by Leonardo Da Vinci for the Milan Cathedral [Codice Atlantico, f.850r], with the representation of the stone blocks clamped together and the texture of the bricks. Normally the surveying drawings, even the most admirable - such as the sections of the Basilica of San Pietro by Ferrabosco (1624) - however complex and accurate, they focus on the architectural detail and not on the constructive aspect. So also, the representations of ruins of the classical era, where references to materials often have an evocative function, referable to the sphere of the picturesque; but certainly Piranesi in the XVIII century draws the construction details of the Roman walls.

[9] From the geometric point of view, the uniqueness of building components deriving from the use of non-industrialized construction processes contrasts with the parametric and standardized logic typical of new buildings. Many experiments deal with the study of procedures for the complex modelling of these geometries [Garagnani 20 I3; Apollonio et al. 2012; Dore, Murphy 2013]. From the information point of view, historic buildings are characterized by a large number of information of various kinds relating to various topics deriving from bibliographic and archival researches (historical documents and photographs, monitoring data, structural information, state of conservation) [Fai et al. 20 I I]. This information, not fully supported by BIM software, must be digitized and connected to digital objects through an expansion of the database to be included in the models [Bruno, Roncella 2019].

[ I0] Oreni et al., 20 I3: underline that the model, to be really useful, must not stop only at the geometric aspects related to the surface of the elements but must extend beyond the surface and must take into consideration stratigraphies, structural aspects and construction rules. In their paper they study the use of BIM for the construction of a library of architectural elements (specifically vaults and slabs) as abacus of local building elements. León-Robles et al., 2018 use BIM to model a stone bridge defining each of the elements that make it up in its construction properties and physical and structural aspects. The project model was used to study the deformations undergone by the bridge from its construction to today and BIM was used for the management and future planning.

[I I] The creation of parametric models of historical construction systems can be carried out through the use of different methodologies depending on: the type of element; the purposes of the modelling; the level of detail to which it is intended (and it is appropriate) to arrive from the geometric point of view and which it is possible to reach from the information point of view; the degree of parameterization and reproducibility that is intended to be conferred on the modelled object. Regarding the intermediate horizontal closures: in the case of a laminated floor with underlying wooden beams, the laminated floor was created within the 'floor' system family and a parametric 'beam system' family was inserted under the floor (fig. 6); in the case of the intermediate vaulted system, on the other hand, the vaulted structural layer was created using a structural floor emptied at the intrados and extrados by a parametric void and, above it, a layered architectural slab was inserted, emptied in correspondence of the parametric vault, by a further parametric void (fig. 9). As for the roof slab with purlins, wooden beams and trusses, a layered 'roof' was created which was completed below by two systems of beams perpendicular to each other with beams of different sections, and by parametric trusses (fig. 7).

[12] The paper has been conceived together by the authors. However, paragraphs can be attributed as follows: S. Brusaporci is the author of the Introduction and Representing the Historical Construction System; A. Tata of the paragraphs Historical Construction System in HBIM Processes and For a parametric Atlas of Historical Construction System; M. Centofanti of the Conclusions.

\section{References}

AAVV (I98I). II recupero dei vecchi centri. Gli aspetti teorici. I modi d'intervento. Atti del Convegno Internazionale di Studi, Udine 22-24 maggio 1981. Udine: Università degli Studi di Udine.

Apollonio Fabrizio Ivan, Gaiani Marco, Zheng Sun (2012). BIM-based modeling and data enrichment of classical architectural buildings. In SCIRES-it, 2, 2012,2 , pp. $41-62$.

Baik Ahmad, Alitany Ayman, Boehm Jan, Robson Stuart (2014). Jeddah Historical Building Information Modelling "JHBIM" Object Library. In ISPRS Ann. Photogramm. Remote Sens. Spatial Inf. Sci., II-5, 20 I 4, pp. 4 I -47.

Bertocci Stefano, Bini Marco (20 I2). Manuale di rilievo architettonico e urbano. Novara: Città Studi.

Bianchini Carlo, Nicastro Saverio (2018). La definizione del Level of Reliability: un contributo alla trasparenza dei processi di Historic-BIM. In EmplerTommaso, Valenti Graziano Mario (a cura di). 3D Modeling \& BIM Nuove Frontiere. Roma: dei Tipografia del genio civile, pp. 208-225.

Bruno Nazarena, Roncella Riccardo (2019). HBIM for conservation: A new proposal for information modelling. In Remote Sensing, 11, 2019, 15, pp. 1751-1774.

Brusaporci Stefano (2007). Le murature dell'architettura del versante meridionale del Gran Sasso (secc. XI-XIV). Roma: Gangemi editore.

Brusaporci Stefano (2017). The Importance of Being Honest: Issues of Transparency in Digital Visualization of Architectural Heritage. In Ippolito Alfonso. Handbook of Research on Emerging Technologies for Architectural and Archaeological Heritage. Hershey, PA: IGI Global, pp. 66-93.

Brusaporci Stefano, Maiezza Pamela,Tata Alessandra (20 I 8a). A Framework for Architectural Heritage HBIM Semantization and Development. In The International Archives of the Photogrammetry, Remote Sensing and Spatial Information Sciences. 20 I 8. XLII-2 pp. $179-184$. 
Brusaporci Stefano, Maiezza Pamela, Tata Alessandra (2018b). Computational Design for As-Built Modeling of Architectural Heritage in HBIM processes. In 2018 IEEE International Conference of Metrology forArchaeology and Cultural Heritage, MetroArchaeo Proceedings. Cassino 22-24 October 2018, pp.194-199.

Brusaporci Stefano, Maiezza Pamela,Tata Alessandra (2019a). Prime riflessioni sulla rappresentazione e parametrizzazione HBIM dell'apparecchiatura costruttiva storica. In Empler Tommaso, Fusinetti Alexandra (a cura di). 3D Modeling \& BIM Modelli e soluzioni per la digitalizzazione. Roma: dei Tipografia del genio civile, pp. I82-197.

Brusaporci Stefano, Maiezza Pamela, Tata Alessandra (2019b). Trasparenza e affidabilità dei modelli HBIM. Transparency and reliability of HBIM models. In Papa Lia Maria, D'Agostino Pierpaolo (a cura di). BIM Views: Esperienze e scenari. Fisciano, SA: CUA pp. $125-140$.

Carbonara Giovanni, Centofanti Mario, Mingucci Roberto (20I5). Disegno per il restauro: oltre il rilievo. In Disegnarecon, n. I4, 20I5, 8, pp. E2.

Carbonara Giovanni (1990). Restauro dei Monumenti. Guida agli elaborati grafici. Napoli: Liguori.

Carbonara Giovanni (1989). Disegnare per il restauro. In Disegnare. Idee Immagini, 0, 1989, pp. 85-94.

Centofanti Mario (20 I0). Della natura del modello architettonico. In Brusaporci Stefano (a cura di). Sistemi informativi integrati per La tutela la conservazione e la valorizzazione del patrimonio architettonico e urbano. Roma: Gangemi, pp. 43-54.

Cetra Valentina (2015). Progetto di restauro di un aggregato nel centro storico di Castel del Monte (AQ). Tesi di Laurea in Ingegneria Edile-Architettura, Università degli Studi dell'Aquila. A.A. 20 I4/20 I5. Relatori: Centofanti Mario, Brusaporci Stefano. Correlatore: Di Fabio Franco.

Curti Mariangela (20I5). Rilievo e restauro del palazzo baronale Falconi in Torre di Taglio (RI). Tesi di Laurea in Ingegneria EdileArchitettura, Università degli Studi dell'Aquila. A.A. 20 I 4/20 I5. Relatore: Centofanti Mario. Correlatore: Brusaporci Stefano.

Di Luggo Antonella, Scandurra Simona, Pulcrano Margherita et al. (2018). Sistemi voltati nei processi cloud to BIM. In Empler Tommaso, Valenti Graziano Mario (a cura di). 3D Modeling \& BIM Nuove Frontiere. Roma: dei Tipografia del genio civile. vol 2 pp. $162-177$.

Di Nucci Anna (2009). L'arte di costruire in Abruzzo. Tecniche murarie nel territorio della Diocesi di Valva e Sulmona. Roma: Gangemi editore.

Docci Mario, Maestri Diego (2009). Manuale di rilevamento architettonico e urbano. Bari: Laterza, pp. 374.

Dore Conor, Murphy Maurice (2013). Semi-Automatic Modelling of Building Façades with Shape Grammars Using Historic Building Information Modelling. In International Archives of the Photogrammetry, Remote Sensing and Spatial Information Sciences, XL-5/WI, pp. 57-64.

Fai Stephen, Graham Katie, Duckworth Todd et al. (201 I). Building Information Modelling and Heritage Documentation. In Fai Stephen, Graham Katie et al. (eds). Conference Proceedings: XXIII CIPA International Symposium. Praga I2- I6 September 20 I I. Praga: CIPA Heritage Documentation.

Fiorani Donatella (1996). Tecniche costruttive murarie medievali. Roma: L'Erma di Bretschneider.

Fiorani Donatella (2004). Restauro Architettonico e strumento informatico. Guida agli elaborati grafici. Napoli: Liguori.

Fiorucci Tiziana, Chiavoni Emanuela (2004). Gli strumenti di conoscenza per il progetto di restauro. Roma: Gangemi editore.

Francovic Riccardo, Parenti Roberto (1988). Archeologia e Restauro dei monumenti. Firenze: L'insegna del Giglio.

Garagnani Simone (2013). Building Information Modeling a real world knowledge. A methodological approach to accurate semantic documentation for the built environment. In DigitalHeritage 20 I 3. Marsiglia: IEEE, pp. 489-496.

Giovanetti Francesco (1990). Manuale del recupero di Città di Castello. Città di Castello: Comune di Città di Castello.

Giuffrè Antonino (1993). Sicurezza e conservazione dei centri storici: il caso Ortigia: codice di pratica per gli interventi antisismici nel centro storico. Roma-Bari: Laterza.

Graziani Enrica M. L. (20I5). Rilievo e Restauro di Palazzo Loddi (XVII sec.) L'Aquila. Tesi di Laurea in Ingegneria Edile-Architettura, Università degli Studi dell'Aquila. A.A. 2014/2015. Relatore: Centofanti Mario. Correlatori: Brusaporci Stefano, Quaresima Raimondo.

León-Robles Carlos A., Reinoso-Gordo Juan F., González-Quiñones Juan J. (2018). Heritage Building Information Modeling (H-BIM) Applied to A Stone Bridge. In ISPRS Int. J. Geo-Information, 2019, 8, p. I21-135.

Logothetis S., Delinasiou A., Stylianidis E. (20I5). Building Information Modelling for Cultural Heritage: A review. In ISPRS Ann. Photogramm. Remote Sens. Spatial Inf. Sci., II-5/W3, 20 I 5, pp. I77-I83.

Maiezza Pamela (2019). As-Built reliability in architectural HBIM modeling. In The International Archives of the Photogrammetry, Remote Sensing and Spatial Information Sciences, 20I9, XLII-2MV9, pp. 46I-466.

Marconi Paolo (1997). Manuale Del Recupero Del Centro Storico Di Palermo. Palermo: Flaccovio, pp. 392.

Marta Roberto (1989). Tecnica Costruttiva a Roma nel medioevo. Roma: Kappa, pp. I 60.

Marta Roberto (I99|). Tecnica Costruttiva Romana. Roma: Kappa. 
Quattrini Ramona, Pierdicca Roberto, Morbidoni Christian (2017). Knowledge-based data enrichment for HBIM: Exploring high-quality models using the semantic-web. In Journal of Cultural Heritage, 28, 20 I 7, pp. I 29- I 39

Murphy Maurice, McGovern Eugene, Pavia Sara (2009). Historic building information modelling (HBIM). In Structural Survey, 27, 4, pp. 3। I-327

Oreni Daniela, Brumana Raffaella, Georgopoulos Andreas et al. (2013). HBIM for Conservation and Management of Built Heritage: Towards a Library of Vaults and Wooden Bean Floors. In ISPRS Annals of the Photogrammetry, Remote Sensing and Spatial Information Sciences, Volume II-5/WI, 20I3, pp. 2I 5-22 I.

Varagnoli Claudio (2000). Materiali per un atlante della costruzione storica in Abruzzo. In Contributi, 7, 2000.

Varagnoli Claudio (2008a). La costruzione tradizionale in Abruzzo. Fonti materiali e tecniche costruttive dalla fine del Medioevo all'Ottocento. Roma: Gangemi editore.

Varagnoli Claudio (2008b). Terre murate. Ricerche sul patrimonio architettonico in Abruzzo e Molise. Roma: Gangemi editore.

Varagnoli Claudio (2009). Muri parlanti. Prospettive per l'analisi e la conservazione dell'edilizia tradizionale. Firenze: Alinea.

Zordan Luigi, Bellicoso Alessandra, De Berardinis Pierluigi et al. (2002). Le tradizioni del costruire della casa in pietra: materiali, tecniche, modelli e sperimentazioni. L'Aquila: GTE.

\section{Authors}

Stefano Brusaporci, Università degli Studi dell'Aquila, stefano.brusaporci@univaq.it.

Alessandra Tata, Università degli Studi dell'Aquila, alessandra.tata@graduate.univaq.it.

Mario Centofanti, Università degli Studi dell'Aquila, mario.centofanti@univaq.it.

To cite this chapter. Brusaporci Stefano, Tata Alessandra, Centofanti Mario (2020). Tecnologie avanzate per la rappresentazione dell'apparecchiatura costruttiva storica: HBIM e il rinnovarsi di una istanza/Advanced technologies for the representation of historical construction systems: HBIM and the renewal of an instance/ln Arena A., Arena M., Brandolino R.G., Colistra D., Ginex G., Mediati D., Nucifora S., Raffa P. (a cura di). Connettere. Un disegno per annodare e tessere. Atti del $42^{\circ}$ Convegno Internazionale dei Docenti delle Discipline della Rappresentazione/Connecting. Drawing for weaving relationships. Proceedings of the 42th International Conference of Representation Disciplines Teachers. Milano: FrancoAngeli, pp. $1778-1799$. 